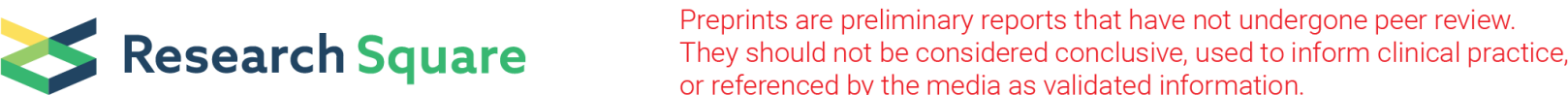 or referenced by the media as validated information.
}

\section{Prehabilitative Resistance Exercise Reduces \\ Neuroinflammation and Improves Mitochondrial \\ Health in Aged Mice with Perioperative \\ Neurocognitive Disorders}

\section{Yan Liu}

The University of Hong Kong

John Man Tak Chu

The University of Hong Kong

You Ran

The University of Hong Kong

\section{Yan Zhang}

The University of Hong Kong

Raymond Chuen Chung Chang

The University of Hong Kong

Gordon Tin Chun Wong ( $\nabla$ gordon@hku.hk)

University of Hong Kong https://orcid.org/0000-0002-8127-4593

\section{Research}

Keywords: Resistance exercise, neuroinflammation, perioperative neurocognitive disorders, mitochondrial function, synaptic deficit

Posted Date: November 2nd, 2021

DOI: https://doi.org/10.21203/rs.3.rs-1010926/v1

License: (c) (i) This work is licensed under a Creative Commons Attribution 4.0 International License. Read Full License 


\section{Abstract \\ Background}

Perioperative neurocognitive dysfunction remains a significant problem in vulnerable groups such as the elderly. While experimental data regarding its possible pathogenic mechanisms accumulates, therapeutic options for this disorder are limited. In this study, we evaluated the neuroprotective effect of a period of preconditioning resistant training on aged mice following abdominal surgery. Further, we examined the underlying mechanism from the perspective of neuroinflammatory state and synaptic plasticity in the hippocampus.

\section{Methods}

18-month-old C57BL/6N mice were trained for 5 weeks using a ladder-climbing protocol with progressively increasing weight loading. Preoperative baseline body parameters, cognitive performance and neuroinflammatory states were assessed and compared between sedentary and trained groups of 9month-old and 18-month-old mice. To access the neuroprotective effect of resistance training on postoperative aged mice, both sedentary and trained mice were subjected to a laparotomy under $3 \%$ sevoflurane anesthesia. Cognitive performance on postoperative day 14, hippocampal neuroinflammation, mitochondrial dysfunction and synaptic plasticity were examined and compared during groups.

\section{Results}

18-month-old mice demonstrated increased body weight, higher peripheral and central inflammatory status, reduction in muscle strength and cognitive performance compared with middle-aged 9-month-old mice, which were improved by resistance exercise. In the laparotomy group, prehabilitative resistant exercise improved cognitive performance and synaptic plasticity, reduced inflammatory factors and glial cells activation after surgery. Furthermore, resistance exercise activated hippocampal PGC$1 a / B D N F / A k t / G S K-3 \beta$ signaling and improved mitochondrial biogenesis, as well as ameliorated mitochondrial dynamics in postoperative aged mice.

\section{Conclusions}

Resistance exercise reduced risk factors for perioperative neurocognitive disorders such as increased body weight, elevated inflammatory markers, and pre-existing cognitive impairment. Accordantly, the preoperative resistance exercise was found to improve surgery-induced adverse effects including cognitive impairment, synaptic deficit and neuroinflammation, possibly by facilitate mitochondrial health through the PGC1-a/BDNF pathway. 


\section{Introduction}

Adverse neurocognitive sequalae following surgery in older patient has been recognized as early as the $1950^{\prime}{ }^{1}$. The recently coined term perioperative neurocognitive disorders (PNDs) include cognitive decline diagnosed before or up to 12 months after surgery ${ }^{2}$. PNDs affect multiple cognitive domains ${ }^{3}$, and are associated with an increased length of hospitalization ${ }^{4}$ and long term dementia ${ }^{5}$. With a global aging population and increasing number of operations performed in the elderly ${ }^{6}$, effective options to mitigate PNDs are urgently required.

Neuroinflammation is closely associated with PNDs as well as other neurodegenerative disease ${ }^{7}$. The hippocampus region is important to cognition and is particularly vulnerable to aging related insults ${ }^{8}$. Chronic neuroinflammation is seen in both aged animals and humans, and the severity of inflammation can be aggravated by surgical trauma, leading to synaptic dysfunction and apoptosis ${ }^{9}$. This biological sequence in the brain is regulated by activated microglia and astrocytes and glial activation can persist and accelerate cognitive decline in susceptible populations. From previous reports, activation of glial cells can lead to subsequent release of pro-inflammatory mediators, which in turn trigger mitochondrial and synaptic dysfunction in various neurodegenerative diseases ${ }^{10}$. In Alzheimer's Disease (AD), activated microglia release inflammatory cytokines and affect mitochondrial dysfunction in the hippocampus ${ }^{11}$. In other reports, targeting the actions of specific pro-inflammatory cytokines, including IL- ${ }^{12}$ and IL- $6^{13}$, have been found to be capable of reducing POCD. Thus, the presence of neuroinflammation correlates with both mitochondrial and synaptic dysregulation and it is possible that reducing neuroinflammation may impede these changes and thus ameliorating cognitive dysfunction in PNDs.

Exercise benefits age-related cognitive changes as well as early-stage dementia ${ }^{14}$, and potentially has a powerful anti-inflammatory effect in neurodegeneration diseases. Specifically, exercise may reduce neuroinflammation, either by attenuating activation of microglia and astrocytes ${ }^{15}$ or by reducing proinflammatory adipokines secretion in crosstalk between muscle and adipose tissue ${ }^{16}$. Physical exercise can regulate innate inflammatory responses via the hypothalamic pituitary adrenal axis and sympathetic nervous system ${ }^{17}$. Moreover, the beneficial effect of physical exercise on neurodegeneration diseases is associated with improving mitochondrial function and genesis ${ }^{18}$.

Aerobic and resistance exercise are two main types of exercise that have been considered to primary target on cardiovascular benefits and muscle hypertrophy respectively ${ }^{19}$. With most studies on the neuroprotective effect of exercise focusing on the aerobic type, the effect of resistance exercise on cognition and synaptic function, particularly in PNDs, remained largely unknown. From a certain point of view, resistance exercise is efficacious and safe for preoperative patients. Resistance type exercises may be performed from a stationary position and theoretically reduces risks of fall in frailer patients. Furthermore, preoperative resistance exercise may increase muscle mass, thus minimizing perioperative muscle loss, that in turn might benefit postoperative rehabilitation. In light of these findings, we hypothesized that preoperative resistance exercise improves perioperative neurocognitive function and 
related neuropathological changes through modulating inflammatory responses and mitochondria dysfunction. An aged mice model was used as advanced age is a risk factor for PNDs and reduced cognitive reserves in the aged may render them more susceptible to the detrimental effects of neuroinflammation.

\section{Materials And Methods}

\subsection{Animal handling, assessment and sacrifice}

Male C57BL6/N mice aged 9-months or 18-months old were housed in the Laboratory Animal Unit at the University of Hong Kong, which is fully accredited by the Association for Assessment and Accreditation of Laboratory Animal Care International. They were first acclimatized for a week to a 12/12 hours' light/dark cycle at temperatures between $20-22^{\circ} \mathrm{C}$ and humidity of $50 \pm 10 \%$, with access to food and water ad libitum. The experimental protocols were approved by the Faculty Committee on the Use of Live Animals in Teaching and Research (CULATR) in The University of Hong Kong (CULATR No. 4192-16). In accordance with the $3 R$ 's, all efforts were made to minimized the number and suffering of mice during experiments.

We first compared 18 months old mice with 9 months old counterparts, dividing them (25 middle-aged mice and 23 aged mice) into sedentary (9M-SED and 18M-SED) and resistance exercised (9M-RE, 18M$\mathrm{RE}$ ) groups, comparing their respective general conditions, muscle strength and cognitive performances. We then randomly divided 18-month old mice into control, laparotomy only (Lap), resistance exercise $(\mathrm{RE})$, and resistance exercise followed by laparotomy (RE+Lap) groups to characterize the effect of resistance exercise on behavioral and pathological changes following surgery. In total, 122 aged mice were used to illustrate the effect of resistance exercise on PNDs. To be specific, 24 mice were used to evaluated early regulation of inflammation and intracellular signal pathways, 66 mice were used for behavioral test, late regulation of inflammation, intracellular signal pathways and Golgi staining, 32 mice were used to investigate changes in mitochondrial markers and morphology. The detailed number of mice used in each group was specified in the figure legends.

The body weights, the weight of the food and water provided for each cage on day 1 and day 3 of each week were recorded at the beginning and the end of the training period. Food and water intake for each mouse was calculated by averaging total consumption of each cage divided by the number of occupants.

After behavioral testing the mice were sacrificed by $\mathrm{CO}_{2}$ asphyxiation in accordance with the guidelines of the American Veterinary Medical Association. Blood samples were collected from the heart before it was perfused with saline. The brain, liver and muscle tissues were subsequently harvested and processed.

\subsection{Resistance training protocol}


Mice were trained according to our previously published protocol using a 1-meter ladder ${ }^{20}$ (Fig. 1 a and 1b). In brief, one week after familiarization with ladder climbing, the mice were trained on alternate days for 4 weeks with a 2 min rest between each climb. They were motivated to climb up 15 times per session, and then progressively heavier weights attached to their tails were introduced. The weights were equivalent to $15 \%, 25 \%, 40 \%$ and $50 \%$ of their body weight, applied at 1, 2, 3 and 4 weeks respectively. The intensity was carefully adjusted based on the individual performances at each exercise session.

Mice became familiarized with the ladder after around 3-4 secessions and those showing natural interest in this training protocol would climb up the ladder spontaneously. During experimentation excessive stress to the mice was minimized by allowing them to first climb up the ladder spontaneously and only motivated them if they stopped in the middle, by a gentle touch to the tail (Additional file 1). Extra rest time was allowed for mice that displayed a refusal to climb or tachypnoea after their 2 minutes rest. If they still refused, the attached weight was progressively reduced by $5 \mathrm{~g}$ until they were willing to climb, then after were gradually reintroduced in subsequent climbs.

\subsection{Strength assessment}

Muscle strength test was conducted following the protocol of Deacon et $\mathrm{al}^{21}$. The mice were held by the middle of the tail and lowered to allow it to grasp a series of weights on the bench with all four paws. The duration of the weight being lifted clear of the table was timed and when successfully held for more than three seconds, the next heavier weight was tested. If the mouse drops the weight within 3 seconds, it was allowed to rest for 20 seconds and then reattempt with the same weight. Testing was completed when the mouse failed to hold the same weight for 3 consecutive attempts. A score was then calculated by the formula: [3 x (heaviest weight $-40 \mathrm{~g})+$ (time held)].

\subsection{Anesthesia and surgery}

A laparotomy was performed under sevoflurane anesthesia following our previous protocol taking approximately 30 minutes $^{22}$. Using a rodent inhalation anesthesia apparatus (Harvard, US), anesthesia was induced with 5-6\% sevoflurane (Sevorane TM, Abbott, Switzerland) in a perspex induction chamber, and maintained at $3-4 \%$ with $0.8 \mathrm{~L} / \mathrm{min}$ oxygen flow. A $2.5 \mathrm{~cm}$ midline incision was made to enter the abdominal cavity. Approximately $10 \mathrm{~cm}$ of the intestine were exteriorized, rubbed vigorously for 1 minute and were left exposed outside the abdominal cavity for a further 2 minutes before replacing it back into the abdominal cavity. Sterile chromic gut sutures (4-0, PS-2; Ethicon, USA) were used to suture the abdominal muscle and the skin in two separate layers. A heating pad was used to keep the mice warm, the rhythm and frequency of respiration, as well as the color of the paw, were monitored.

\subsection{Behavioral and cognitive assessment}

The behavioral tests were performed from least-to-most stressful order to minimize possible interference by any residual distress from the previous test. They were conducted in a dedicated room with no natural light, noise, odor, or other animals not involved with testing present. While still in their home cages the mice were acclimatized to this environment for 30 minutes. Before each round the experimental 
apparatus was cleaned using $70 \%$ ethanol to remove residual odors. The mice were tested in a random order between and within groups.

\subsubsection{Open field test}

The open field test makes use of rodents' natural aversion to open spaces to assess general locomotor activity and anxiety of rodents ${ }^{23}$ and was performed on postoperative day 12 using an enclosed gridded arena of $40 \mathrm{~cm} \times 40 \mathrm{~cm} \times 40 \mathrm{~cm}$ under dim lighting. The floor of the box was demarcated into 9 squares, each measuring approximately $13.3 \mathrm{~cm} \times 13.3 \mathrm{~cm}$, with the middle one designated as the central area. Each mouse was gently placed in that middle square and allowed to spontaneously explore the arena without any interference. This spontaneous activity of the mouse was video recorded for 10 minutes. The total exploration time in the central area indicates anxiety, whereas the frequency of crossing grid lines is a measure of locomotor activity.

A video tracking software, SMART 3.0 (Panlab SL) were used for data analysis following the operation manual. Specifically, the arena was equally divided into 9 zones (as showed in Fig. 3b), the total exploration time of mice in the central area of $13.3 \times 13.3 \mathrm{~cm}^{2}$ indicates anxiety/depression, and the total transition during different zones, total distance travelled and mean speed are measures of locomotor activity.

\subsubsection{Novel Object Recognition test}

The NOR test exploits a rodent's preference to explore a novel rather than a familiar object to measure the animal's recognition memory and exploration tendencies ${ }^{24}$ and was performed 24 hours after the open field test. Two identical objects $(A+A)$ were placed at opposite corners in the arena and the mice were allowed to explore them freely for 10 minutes. Following a 6-hour retention interval, the animals were placed back to the arena, this time with one object being the identical $(A)$ and the other being novel $(B)$, with the two objects situation in the same position in the arena. We alternated the location of novel versus familiar object for each batch of tests. An interaction was defined if the nose of the mouse points to the object within $2 \mathrm{~cm}$. The discriminating index is the ratio of the time exploring the novel object over the total time spent exploring both objects.

\subsubsection{Y-maze test}

Y-maze test was performed following a previous published protocol ${ }^{25}$. The Y-maze test was designed to assess aversive memory and hippocampal-dependent spatial learning. The apparatus consists of three interconnected plastic compartments angled at 120 degrees from each other to shape like the capital letter " $Y$ ", with each compartment measuring $32 \mathrm{~cm}$ (long) $\times 10 \mathrm{~cm}$ (wide) $\times 10 \mathrm{~cm}$ (high). One of the compartments is white with a transparent cover, while the remaining two are black with an opaque cover. The floor of the black arms is lined with $3.2 \mathrm{~mm}$ stainless steel rods placed $8 \mathrm{~mm}$ apart capable of delivering electric shocks $(2 \mathrm{~Hz}, 10 \mathrm{~s}, 40 \pm 5 \mathrm{~V})$. During the preoperative training, each mouse was allowed to explore the three arms freely for 5 minutes. Thereafter the animal was placed in the blind end of one of 
the black arms randomly. Electric shocks were applied until the animal enters the shock-free white compartment. A correct choice was recorded when the animal enters the white compartment within 10 seconds and stays there for at least 30 seconds. An error occurs when the animal enters the other black compartment or failed to enter and stay in the white compartment within the appropriate time. Successful training was defined as 9 consecutive correct choices. Postoperatively each mouse was tested 10 times and the number of errors and time before entering the shock-free compartment (latency) were recorded.

\subsection{Quantitative Real-time Polymerase Chain Reaction (PCR)}

Under RNase-free conditions, the brain and liver tissues were separately homogenized using Tri-Reagent ${ }^{\circledR}$ (MRC, USA). The RNA quality was assessed by optical density (OD) measurement (Nanodrop1000; Thermo Fisher Scientific, USA). Only isolated RNA samples with an OD 260/280 ratio > 1.8 and OD $260 / 230$ ratio $<2.0$ were used for analysis. Isolated RNA was further purified by removing genomic DNA with an Ambion ${ }^{\circledR}$ DNA-freeTM DNA Removal Kit (Invitrogen, USA), then underwent reverse transcription using PrimeScriptTM Master-Mix Kit (TAKARA, Japan). PCR was performed using StepOnePlusTM RealTime PCR system (Applied Biosystems, USA) with the SYBR® Premix Ex-TaqTM II Kit (TAKARA, Japan). The amplification conditions were $95^{\circ} \mathrm{C}$ for $20 \mathrm{~s}$, followed by 40 cycles of denaturation at $95^{\circ} \mathrm{C}$ for $15 \mathrm{~s}$, extension at different gene-specific annealing temperature as described in table 1 in additional file 2 (which also included primer sequences), and data capture was conducted at $72^{\circ} \mathrm{C}$ for 30 s. The relative levels of cytokines were normalized to those of the endogenous reference glyceraldehyde-3-phosphate dehydrogenase (GAPDH) following the $2^{-\triangle \Delta C t}$ method.

\subsection{Western Blot}

Total lysates or cellular compartment fractions were prepared using RIPA buffer or commercial kit (procedure detailed in additional file 2 ), subjected to $10 \%, 12.5 \%$, or $15 \%$ polyacrylamide gels electrophoresis depending on the molecular weight of target protein, and transferred onto PVDF membranes. Non-specific binding sites were blocked with $5 \%$ non-fat milk for 1 hour at room temperature. Then the membranes were incubated overnight at $4^{\circ} \mathrm{C}$ with specific primary antibodies. After the incubation with horseradish peroxidase-conjugated secondary antibodies (DAKO, Denmark) for 2 hours, the immunoreactive band signal intensity was subsequently visualized by chemiluminescence (WesternBrightTM ECL, or WesternBrightTM Quantum). Signals were captured by ChemiDoc ${ }^{\text {TM }}$ Touch Imaging System (Bio-Rad, USA). Immunoblots were normalized for gel loading with $\beta$-actin or GAPDH antibodies (Sigma-Aldrich, USA). The intensities of chemiluminescent bands were measured by Image Lab $^{\text {TM }}$ Touch Software Version 1.2 (provided by Bio-Rad, USA). Primary and secondary antibodies used were shown in table 2 in additional file 2.

\subsection{Immunofluorescent Staining}

Twenty-micron thick coronal sections were cut for immunofluorescent staining following a previous protocol ${ }^{26}$ (sections in $30 \mu \mathrm{m}$ thickness were used for Iba-1 and GFAP staining. After antigen retrieval with $0.01 \mathrm{M}$ citrate buffer ( $\mathrm{pH} 6.0$ ) at $90^{\circ} \mathrm{C}$ for 15 minutes), $5 \%$ normal goat serum was used for blocking 
non-specific binding. The sections were then incubated with specific primary antibodies at $4^{\circ} \mathrm{C}$ overnight. Sections were then incubated with specific Alexa Fluor 568 or 488 secondary antibodies (Invitrogen, USA) for 2 hours at room temperature (table $2 \mathrm{~b}$ in additional file 2 ). The counterstaining was performed with 3 $\mu \mathrm{M}$ 4'-6-diamidino-2-phenylindole (DAPI). Immunolabeled tissues were observed under a laser scanning confocal fluorescent microscope (Carl Zeiss LSM 700, Germany) equipped with ZEN light software at $1024 \times 1024$ resolution, Z-stack images were acquired. All quantitative analyses were performed on at least three images per animal from three independent experiments. We followed Tavares et al' methods 27 to perform the morphological analysis of astrocytes using Simple Neurite Tracer plugin of Image J. The investigator conducting data analysis was blinded to minimize analytical bias.

\subsection{Golgi-staining}

Whole brains were immediately removed without perfusion and rinsed with double distilled water for 2-3 seconds to remove any blood on its surface. Hito Golgi-Cox OptimStain Kit (Hitobiotec Inc., Wilmington, $D E, ~ U S A)$ was used for the tissue preparation and staining procedure, conducted in strict adherence to the manufacturer's user manual and material safety data sheet. A series of $150 \mu \mathrm{m}$ thick coronal sections were sliced from the hippocampus using a cryostat (Leica, Wetzlar, Germany). Pictures were taken of the hippocampal DG region of each brain. Dendritic branching was assessed using serial photographs taken with a 20x objective lens that provides sufficient dendritic branching resolution. Sholl analysis was conducted using a Sholl analysis plug-in (http://fiji.sc/Sholl Analysis) for Image J software (National Institutes of Health, Bethesda, MD, USA) to demonstrate dendritic complexity.

Spine density was analyzed using serial photographs taken with $100 \times$ objective lens. Only neurons with well impregnated dendritic trees, intact cell bodies and dendrites in full view in the plane of section and not obscured by other blood vessels, astrocytes, or dendritic clusters were analyzed. Dendritic spine density of the DG was measured. For each cell, $20 \mu \mathrm{m}$ long segments of apical densities on the lower half of the apical segments were traced at 1000x magnification. The number of spines was recorded and together with the length of the dendritic segment the number of spines per $\mu \mathrm{m}$ was calculated. No corrections were made for spines obscured by the overlying dendrites, so the data obtained might be an underestimate of the actual density. The morphology analysis of neurons was performed by a blinded assessor.

\subsection{Transmission electron microscopy (TEM)}

A Philips CM100 TEM was used to demonstrate the morphological changes in the mitochondria. Briefly, hippocampi were rapidly isolated and cut into $2 \mathrm{~mm}$ cubes followed by fixation in $2.5 \%$ glutaraldehyde (EM grade) in $0.1 \mathrm{M}$ buffer overnight and then processed to slice by professional technicians in the Electron Microscope Unit of Queen Marry Hospital, the University of Hong Kong. To demonstrate mitochondria in the DG, the nucleus of DG granule cells was first identified under 700x, and photos with higher magnification focused on granule cells project zones were taken, which captured mitochondria both near the synapse and away from the nucleus. For each slice, 2200x and 3900x pictures were taken to analyze mitochondrial density and abnormalities, and ER-mitochondria contacts were analyzed using 
11500x pictures to demonstrate the ultrastructure. The subsequent number and morphology analysis of mitochondria was performed using Image $\mathrm{J}$ by a blinded assessor. Abnormal mitochondria are characterized by irregular, swollen or whorling cristae, swollen outer or discontinuous inter or outer membranes ${ }^{28}$, the typical morphology of abnormal mitochondria were shown in Fig. $7 \mathrm{~g}$.

\subsection{Statistical analysis}

All data were first examined by the Shapiro-Wilk normality test using Prism 7.0 (Graphpad Software, USA) to check for normal distribution. A repeated-measured ANOVA was used to analyze the data obtained for the food and water intake. Either paired or unpaired two-tailed Student's t-test was used to analyze the data obtained for body weight, muscle strength and RT-PCR. A two-way ANOVA was used for statistical analysis of parametric data for all the rest data, including behavioral test, Golgi staining, Immunofluorescent staining, western blot and TEM. All results were presented as the means \pm standard error of the mean (SEM). In all cases, a p-value of less than 0.05 was considered statistically significant.

\section{Results}

\subsection{General conditions and pre-existing cognitive impairment in aged mice was improved by resistance exercise}

Mice were trained using a 1-meter ladder (Fig. 1a), body weight, food and water intake, as well as muscle strength were monitored during resistance training (Fig. 1b). Food and water intake did not differ between the active and sedentary groups (Fig. 1c). Aged mice were on average heavier than middle-aged mice, showing by an increased body weight baseline $(5.23 \pm 2.1 \mathrm{~g}, p=0.0205$, Fig. 1$)$. The body weight of both middle-aged and aged sedentary mice was significantly increased after 5 weeks $(3.71 \pm 0.5 \mathrm{~g}, p<0.0001$ for $9 \mathrm{M}$ mice; $4.31 \pm 0.5 \mathrm{~g}, p<0.0001$ for $18 \mathrm{M}$ mice, Fig. $1 \mathrm{~d}=$. Resistance exercise reduced the body weight only in middle-aged but not older mice $(-2.47 \pm 0.65 \mathrm{~g}, p=0.0024$ for middle-aged mice; $p=0.3002$ for aged mice, Fig. 1d), nevertheless, resistance exercise prevented weight gain otherwise seen in sedentary aged mice (interaction: $\mathrm{F}_{(1,44)}=1.008, p=0.3209$; age factor: $\mathrm{F}_{(1,44)}=4.132, p=0.0481$; exercise factor: $F_{(1,44)}=90.59, p<0.0001$. Multiple comparisons: $-5.0 \pm 0.85 \mathrm{~g}, p<0.0001$ for aged mice, Fig. 1e).

Recent studies suggest muscle strength can be used as an index to monitor progression of cognitive decline in old adults ${ }^{29}$. Aged mice have significantly lower baseline muscle strength compared with their younger counterparts $(-7.27 \pm 1.46$ points, $p<0.0001$, Fig. 1f). Five weeks of resistance training significantly improved the weight lifting performance in mice from both groups (3.54 \pm 1.39 points, $p=$ 0.0256 for $9 \mathrm{M}$ mice; $7.99 \pm 1.18$ points, $p<0.000118 \mathrm{M}$ mice, Fig. $1 \mathrm{f}=$. A similar trend was seen in further analysis of muscle strength change (interaction: $F_{(1,43)}=0.5549, p=0.4604$; age factor: $F_{(1,43)}=7.468$, $p=0.0091$; exercise factor: $\mathrm{F}_{(1,43)}=25.71, p<0.0001$. Multiple comparisons: $5.538 \pm 1.794, p=0.0179$ 
for $9 \mathrm{M}$ mice; $7.446 \pm 1.828, p=0.0011$ for $18 \mathrm{M}$ mice, Fig. $1 \mathrm{~g})$. The effect of resistance exercise on learning and memory was evaluated by the $Y$ maze test. We found a significant interaction between age and exercise, suggesting that the effect of resistance exercise differed between middle-aged mice and aged mice (for errors, interaction: $\mathrm{F}_{(1,44)}=6.497, p=0.0144$; age factor: $\mathrm{F}_{(1,44)}=46.59, p<0.0001$; exercise factor: $F_{(1,44)}=10.05, p=0.0028$, Fig. 1h. For latency, interaction: $F_{(1,44)}=2.301, p=0.1364$, age factor: $F_{(1,44)}=38.46, p<0.0001$; exercise factor: $F_{(1,44)}=5.838, p=0.0199$, Fig. 1i). Multiple comparisons found that aged mice demonstrated deficits in aversive memory and hippocampaldependent spatial learning (errors: $5.1 \pm 0.79, p<0.0001$, Fig. $1 \mathrm{~h}$; latency: $4.03 \pm 0.75 \mathrm{~s}, p<0.0001$, Fig. 1i) when compared with the middle-aged mice. Resistance exercise reduced both errors $(-3.1 \pm 0.79$, $p=0.0015$, Fig. $1 \mathrm{~h})$ and latency $(-2.05 \pm 0.75 \mathrm{~s}, p=0.0439$, Fig. 1i) in aged mice.

Therefore, the current training protocol prevented the aged mice from becoming overweight without affecting their food and water intake, improved their muscle strength and cognitive function. Given that obesity ${ }^{30}$ and pre-existing cognitive impairment ${ }^{31}$ are independent risk factors for PNDs, our data indicate that resistance exercise might reduce the incidence of postoperative cognitive decline.

\subsection{Resistance training improved postoperative cognitive performance}

A laparotomy was performed $24 \mathrm{~h}$ after the last resistance training session and the open field test and NOR tests were conducted on postoperative days 12 and 13(see Fig. 2a). As can be seen from figures $2 b$ $\& 2 \mathrm{c}$, postoperative locomotor activity was not affected in any of the groups. Surgery induced anxiety in mice from the laparotomy only group as indicated by a decreased central duration compared with control (interaction: $\mathrm{F}_{(1,62)}=1.347, p=0.2502$; laparotomy factor: $\mathrm{F}_{(1,62)}=7.808, p=0.0069$; exercise factors: $\mathrm{F}$ $(1,62)=6.119, p=0.0161$. Multiple comparisons: $-54.93 \pm 19.04 \mathrm{~s} ; p=0.0268$ compared to control, Fig. 2d). Pre-operative resistance exercise appears to exert some anxiolytic effects, but the increased central duration time in the RE + Lap group failed to reach statistical significance $(p=0.0585$ compared to laparotomy group, Fig. 2d).

Mice from the laparotomy group demonstrated the presence of recognition memory deficits following surgery as indicated by the significant decrease in discrimination index in the NOR test (interaction: $\mathrm{F}_{\text {(1, }}$ $62)=3.449, p=0.0680$; laparotomy factor: $\mathrm{F}_{(1,62)}=11.6, p=0.0012$; exercise factor: $\mathrm{F}_{(1,62)}=6.158, p=$ 0.0158 . Multiple comparisons: $-0.16 \pm 0.04, p=0.0016$ compared to control, Fig. $2 \mathrm{e})$. Similarly, they displayed more errors (interaction: $\mathrm{F}_{(1,62)}=14.01, p=0.0004$; laparotomy factor: $\mathrm{F}_{(1,62)}=5.778, p=$ 0.0192; exercise factor: $\mathrm{F}_{(1,62)}=8.049, p=0.0061$. Multiple comparisons: $2.56 \pm 0.57, p=0.0002$. Fig. 2f) and longer escape latency (interaction: $\mathrm{F}_{(1,62)}=1.65, p=0.2037$; laparotomy factor: $\mathrm{F}_{(1,62)}=6.092, p=$ 0.0164; exercise factor: $F_{(1,62)}=6.846, p=0.0111$. Multiple comparisons: $1.83 \pm 0.67 \mathrm{~s}, p=0.0392$. Fig. $2 \mathrm{~g}$ ) compared to control in the Y-maze test. Preoperative resistance training attenuated these changes when compared to laparotomy only mice (discrimination index: $0.13 \pm 0.04, p=0.0162$; errors: $-2.7 \pm 0.6, p<0.0001$; latency: $-1.9 \pm 0.7 \mathrm{~s}, p=0.0368$, Fig. 2e-g). 


\subsection{Resistance training attenuated the decreased in dendritic process complexity and spine density following laparotomy}

To evaluate whether the beneficial effect of resistance exercise on postoperative cognitive function was accompanied by structural improvements in neurons, we used Golgi-staining to show the morphology of dendrites and spines (Fig. 3a \& 3b). The spine density was significantly decreased in the surgery group (interaction: $\mathrm{F}_{(1,68)}=1.89, p=0.1738$; laparotomy factor: $\mathrm{F}_{(1,68)}=10.96, p=0.0015$; exercise factor: $\mathrm{F}_{(1 \text {, }}$ $\left.{ }_{68}\right)=7.189, p=0.0092$. Multiple comparisons: $-10.63 \pm 3.21 \%, p=0.0079$, compared to control, Fig. $\left.3 \mathrm{c}\right)$. Preoperative resistance training ameliorated this reduction $(9.21 \pm 3.21 \%, p=0.0275$, Fig. $3 c)$. Sholl profiles generated by semi-automated analysis showed a reduction in dendritic crossings following surgery, indicative of decreased dendritic length and branching within $145-200 \mu \mathrm{m}$ of the radius (interaction: $\mathrm{F}_{(150,2700)}=1.833, p<0.0001$; laparotomy factor: $\mathrm{F}_{(50,2700)}=194.5, p<0.0001$; exercise factor: $\mathrm{F}_{(3,54)}=7.736, p=0.0002$. Multiple comparisons: $p<0.05, p<0.01$, compared to control, Fig. $3 \mathrm{~d}-$ f). Resistance exercise group had greater numbers of dendritic crossings within $115-210 \mu \mathrm{m}$ of the radius compared to laparotomy group $(p<0.05, p<0.01, p<0.001, p<0.0001$, compared to laparotomy group, Fig. 3d-f). Combining with the behavioral data, our result indicates that resistance exercise might be an effective strategy to prevent PNDs, as well as surgery-induced dendritic and synaptic deficit.

\subsection{Preoperative pro-inflammatory disposition in aged mice and early inflammatory responses were reduced by resistance exercise}

Both animal and human data suggest that ageing is commonly associated with chronic neuroinflammation "inflammaging", which can be acute inflammation such as that following surgery, and thereby causing neural dysfunction manifesting as PNDs ${ }^{9}$. The immediate effect of resistance exercise on peripheral and central inflammatory cytokines were analyzed using tissues (hippocampus, muscle and liver) obtained $30 \mathrm{~min}$ after the last resistance training session. Dynamic changes in the expression of inflammatory cytokines (TNF-a, IL-1 $\beta$ and IL-10) were demonstrated in the hippocampus (IL-10: $0.78 \pm$ $0.22, p=0.0048$; Fig. 4a), muscle (IL-1 $\beta$ : $1.58 \pm 0.61, p=0.0276$; IL-10: $6.7 \pm 2.4, p=0.0193$; Fig. 4a) and liver (IL-1 $\beta$ : $-0.42 \pm 0.18, p=0.0406$; TNF-a: $-0.59 \pm 0.14, p=0.0022$; Fig. $4 a$ ). In conjunction with a reduction in pro-inflammatory cytokines in the liver, as well as the elevated levels of IL-10 in the hippocampus and muscle, resistance training induced an anti-inflammatory tendency both peripherally and in the CNS.

On postoperative day 1 , we observed a significant increase in the mRNA expression of MCP-1 in the hippocampus of surgical mice compared to that of control (Interaction: $F_{(1,28)}=4.531, p=0.0422$; laparotomy factor: $F_{(1,28)}=5.756, p=0.0233$; exercise factor: $F_{(1,28)}=6.207, p=0.0189$. Multiple comparisons: $1.16 \pm 0.36, p=0.017$, Figure $4 \mathrm{~b})$. Resistance exercise reduced the expression of MCP-1 in 
the hippocampus $(-1.18 \pm 0.36, p=0.014$, Figure $4 b)$, no difference was seen in the expression of IL$1 \beta, T N F-a$ and IL-10 (Fig. 4b).

\subsection{Resistance exercise alleviates prolonged inflammatory response following surgery}

The effect of resistance exercise on the sustained postoperative inflammatory response was examined on postoperative day 14. Microglia play a key role in the neuroinflammatory response in the brain and the number of Iba-1 label positive microglia was increased in the CA1 region of the hippocampus compared to control (interaction: $\mathrm{F}_{(1,16)}=6.578, p=0.0208$; laparotomy factor: $\mathrm{F}_{(1,16)}=12.57, p=0.0027$; exercise factor: $F_{(1,16)}=21.87, p=0.0003$. Multiple comparisons: $16.2 \pm 3.8, p=0.0032$; Fig. 5a). Activated microglia with hypertrophic cell body were also present in the CA3 region of the hippocampus postoperatively compared to control (interaction: $\mathrm{F}_{(1,16)}=3.053, p=0.0997$; laparotomy factor: $\mathrm{F}_{(1,16)}=$ 7.591, $p=0.0141$; exercise factor: $\mathrm{F}_{(1,16)}=18.23, p=0.0006$. Multiple comparisons: CA3: $0.33 \pm 0.10, p=$ 0.0266 , Fig. $5 b)$. Preoperative resistance training attenuated this microglial activation by reducing their number (CA1: $-19.2 \pm 3.8, p=0.0005$, Fig. $5 a)$ as well as cell body size (CA3: $-0.44 \pm 0.10, p=0.0031$, Fig. 5b) compared to the laparotomy only group.

Astrocytes has been found to mediate microglial activation in surgery-induced neuroinflammation through MCP-1-CCR2 signaling ${ }^{32}$. GFAP staining revealed a notable astrocyte proliferation in the DG region following surgery, with an increase in GFAP intensity (interaction: $F_{(1,12)}=3.753, p=0.0766$; laparotomy factor: $\mathrm{F}_{(1,12)}=5.755, p=0.0336$; exercise factor: $\mathrm{F}_{(1,12)}=6.664, p=0.0240$. Multiple comparisons: $0.84 \pm 0.28, p=0.043$, Fig. $5 e$ ) and cell volume (interaction: $F_{(1,76)}=2.832, p=0.0965$; laparotomy factor: $F_{(1,76)}=9.053, p=0.0036$; exercise factor: $F_{(1,76)}=7.513, p=0.0076$. Multiple comparisons: $54.6 \pm 16.5, p=0.0075$; Fig. $5 \mathrm{~h}$ ) compared to control. Preoperative resistance exercise ameliorated these changes compared to surgery alone (GFAP intensity: $-0.88 \pm 0.28, p=0.034$; cell volume: $-51.58 \pm 16.46, p=0.013$, Fig. $5 d-h)$.

\subsection{Alterations in myokines and Intracellular signal pathways following resistance exercise and surgery}

Various forms of exercise, particularly of the aerobic type, have been shown to trigger production of myokines that can reduce neuroinflammation ${ }^{33}$ and confer positive effect in cognition ${ }^{34}$. PGC- $1 \mathrm{a}$ is an important regulator of mitochondrial biogenesis, and it is known that Bcl-2 and Bax regulate the mitochondria-related intrinsic apoptosis by regulating the permeabilization of mitochondrial membrane, cytochrome $\mathrm{C}$ release and mitochondrial function ${ }^{35}$. The immediate effect of resistance exercise on myokines were analyzed by using tissues obtained $30 \mathrm{~min}$ after the last resistance training trail. The data showed that resistance training increased levels of myokines in the muscle (FGF-21: $5.38 \pm 1.57, p=$ 0.0066; IL-6: $6.62 \pm$ 1.49, $p=0.0012$; Fig. 6a), liver (FGF21: $1.97 \pm 0.90, p=0.0537$; PGC1-a: $0.788 \pm$ $0.3371, p=0.0415$; IL-6: $1.141 \pm 0.4779, p=0.0381$; Fig. 6b), and hippocampus (FGF-21: $2.58 \pm 0.92, p=$ 0.0188; PGC1-a: $0.52 \pm 0.22, p=0.0405$; Fig. 6c) as measured by RT-PCR. 
The expression of BDNF/Akt/GSK3 $\beta$ and Bax/Bcl-2 were evaluated at 24 hours and 14 days after surgery, respectively. There was a decrease in BDNF (interaction: $\mathrm{F}_{(1,20)}=3.59, p=0.0727$; laparotomy factor: $\mathrm{F}_{(1 \text {, }}$ 20) $=5.325, p=0.0318$; exercise factor: $\mathrm{F}_{(1,20)}=19.31, p=0.0003$. Multiple comparisons: $-0.408 \pm 0.144$, $p=0.035$, Fig. $6 \mathrm{e}$ ) and phospho-Akt at serine 473 (interaction: $\mathrm{F}_{(1,20)}=9.614, p=0.0056$; laparotomy factor: $\mathrm{F}_{(1,20)}=10.15, p=0.0046$; exercise factor: $\mathrm{F}_{(1,20)}=3.137, p=0.0918$. Multiple comparisons: $-0.463 \pm 0.104, p=0.0013$, Fig. $6 \mathrm{e})$ in the laparotomy group when compared to controls. Accordingly, surgery significantly increased the Bax/Bcl-2 ratio compared to control (interaction: $\mathrm{F}_{(1,20)}=4.66, p=$ 0.0432, laparotomy factor: $\mathrm{F}_{(1,20)}=18.44, p=0.0004$; exercise factor: $\mathrm{F}_{(1,20)}=5.131, p=0.0348$. Multiple comparisons: $-1.914 \pm 0.42, p=0.001$, Fig. 6f) and prior resistance exercise attenuated these change (BDNF: $0.641 \pm 0.144, p=0.001$; p-Akt: $0.359 \pm 0.104, p=0.0126$, p-GSK3B: $0.6764 \pm 0.1613, p=$ $0.0023 ; \mathrm{Bax} / \mathrm{Bcl}-2$ ratio: $-1.312 \pm 0.42, p=0.021$, Fig. $6 \mathrm{~d}-\mathrm{f}$ ) compared to the laparotomy group.

The changes in these markers were on day 14 similar except for phospho-Akt at serine 473 where no change was seen. The expression of BDNF (interaction: $\mathrm{F}_{(1,20)}=4.673, p=0.0429 ; \mathrm{F}_{(1,20)}=7.709, p=$ $0.0116 ; \mathrm{F}_{(1,20)}=4.937, p=0.0380$. Multiple comparisons: $-0.6325 \pm 0.1811, p=0.0113$, Fig. $\left.6 \mathrm{~h}\right)$ and phospho-GSK3 $\beta$ at serine 9 (interaction: $\mathrm{F}_{(1,20)}=1.229, p=0.2808$; laparotomy factor: $\mathrm{F}_{(1,20)}=9.424, p$ $=0.0060$; exercise factor: $F_{(1,20)}=16.06, p=0.0007$. Multiple comparisons: $0.387 \pm 0.131, p=0.0362$. Fig. $6 \mathrm{~h})$ was decreased. The Bax/Bcl-2 ratio was similarly increased $\left(F_{(1,20)}=15.83, p=0.0007\right.$; laparotomy factor: $\mathrm{F}_{(1,20)}=2.735, p=0.1138$; exercise factor: $\mathrm{F}_{(1,20)}=11.5, p=0.0029$. Multiple comparisons: $0.774 \pm 0.194, p=0.004$. Fig. $6 \mathrm{i})$. Resistance exercise prior to surgery restored these changes to that comparable to the control (BDNF: $0.562 \pm 0.181, p=0.027$; phospho-GSK3 $\beta$ at serine 9: $0.4739 \pm 0.131, p=0.0086 ; \mathrm{Bax} / \mathrm{Bcl}-2$ ratio: $1.012 \pm 0.1943, p=0.0002$, Fig. $6 \mathrm{~g}-\mathrm{i})$.

\subsection{Resistance exercise restored surgery-induced deficits in mitochondria density and morphology}

Our data demonstrated a significant decrease in HSP60 in cytosolic fraction from the laparotomy group (interaction: $F_{(1,20)}=5.291, p=0.0323$; laparotomy factor: $F_{(1,20)}=5.194, p=0.0338$; exercise factor: $F$ $(1,20)=8.106, p=0.010$. Multiple comparisons: $-0.5908 \pm 0.1825, p=0.0198$, Fig. 7b). Importantly, a significant increase of cytochrome $\mathrm{C}$ was seen in the cytosolic fraction following surgery (interaction: $\mathrm{F}_{(1}$, $20)=6.955, p=0.0158$; laparotomy factor: $\mathrm{F}_{(1,20)}=18.45, p=0.0004$; exercise factor: $\mathrm{F}_{(1,20)}=4.514, p=$ 0.0463. Multiple comparisons: $0.951 \pm 0.194, p=0.0005$; Fig. $7 \mathrm{c}$ ), both of which could be reduced by prior resistance exercise (RE + Lap compared to Lap, HSP60: $-0.6641 \pm 0.1825, p=0.0082$; cytochrome C: $-0.6533 \pm 0.194, p=0.0149$, Fig. $7 \mathrm{~b}$ and $\mathrm{c}$ ). There was no change in relative levels of HSP60 or cytochrome $\mathrm{C}$ in the mitochondria fractions (Fig. $7 \mathrm{~b}$ and $\mathrm{c}$ ).

Transmission electron microscopy (TEM) demonstrated a decreased in the total number of mitochondria after laparotomy (interaction: $\mathrm{F}_{(1,172)}=7.266, \mathrm{p}=0.0077$; laparotomy factor: $\mathrm{F}_{(1,172)}=3.88, p=0.0505$; exercise factor: $F_{(1,172)}=8.03, p=0.0052$. Multiple comparisons: $-0.3068 \pm 0.9301, p=0.0064$; Fig. $7 d$ and e), which was not seen in the RE + Lap group (RE + Lap compared to Lap: $0.3636 \pm 0.9301, p=$ 
0.0008; Fig. 7d and e). Although there was no significant difference between the control and surgery groups, resistance exercise significantly reduced the percentage of abnormal mitochondria (interaction: $\mathrm{F}$ $(1,172)=3.039, p=0.0831$; laparotomy factor: $\mathrm{F}_{(1,172)}=0.7192, p=0.3976$; exercise factor: $\mathrm{F}_{(1,172)}=$ 8.117, $p=0.0049$. Multiple comparisons: $-0.7502 \pm 0.023 \%, p=0.0076$; Fig. $7 f-h$ ).

\subsection{Effect of resistance exercise and surgery on mitochondria dynamics.}

Mitochondria undergo 'mitochondrial dynamics', which are critical for various cellular processes including inflammation, apoptosis and cell cycle, as well as mitochondrial quality control. We evaluated several important mitochondrial dynamic markers, including mitofusin-1, mitofusin-2, dynamin related protein 1 (Drp-1) and optic atrophy 1 (OPA1). Surgery-induced a significant increase of mitofusin-2 (interaction: $F_{(1,}$ 28) $=8.425, p=0.0071$; laparotomy factor: $\mathrm{F}_{(1,28)}=14.28, p=0.0008$; exercise factor: $\mathrm{F}_{(1,28)}=1.596, p=$ 0.2169 . Multiple comparisons: $0.8668 \pm 0.1835, p=0.0004$; Fig. $8 \mathrm{~b})$, and this upregulation was reduced by resistance exercise $(-0.5405 \pm 0.1835, p=0.0379 ; \mathrm{Fig}$. $8 \mathrm{~b})$. No changes were demonstrated for the other markers. Mitofusin-2 is responsible for tethering endoplasmic reticulum to mitochondria (ERmitochondria contacts) that allow $\mathrm{Ca}^{2+}$ influx from the ER to mitochondria and these were increased in the Lap (interaction: $\mathrm{F}_{(1,76)}=9.632, p=0.0027$; laparotomy factor: $\mathrm{F}_{(1,76)}=12.4, p=0.0007$; exercise factor: $F_{(1,76)}=14.4, p=0.0003$. Multiple comparisons: $-0.7114 \pm 0.1519, p<0.0001$, Fig. $\left.8 \mathrm{c} c-d\right)$ but not in the RE + Lap group $(-0.7407 \pm 0.1519, p<0.0001$, Fig. $8 c-d=$.

Synapses are sites of high-energy demand that are particularly vulnerable to mitochondria dysfunction. Synaptosomes were isolated from the hippocampus and there was a significant increase of phosphoNR2A at tyrosine 1246 (interaction: $F_{(1,17)}=8.946, p=0.0082 ; F_{(1,17)}=12.49, p=0.0026 ; F_{(1,17)}=$ $1.451, p=0.2449$. Multiple comparisons: $-4.422 \pm 0.9373, p=0.0010$; Fig. $8 \mathrm{e}$ and $\mathrm{f}$ ) and phospho-NR2B at tyrosine 1472 (interaction: $\mathrm{F}_{(1,17)}=6.592, p=0.0200$; laparotomy factor: $\mathrm{F}_{(1,17)}=2.191, p=0.1571$; exercise factor: $F_{(1,17)}=3.088, p=0.0969$. Multiple comparisons: $1.547 \pm 0.5286, p=0.0424$, Fig. $8 \mathrm{e}$ and f) following surgery. The level of phospho-AMPK-a at Thr172, which can be activated by excessive NMDA receptors activity, was enhanced following surgery (interaction: $F_{(1,17)}=6.955, p=0.0173$; laparotomy factor: $F_{(1,17)}=1.919, p=0.1838, F_{(1,17)}=4.573, p=0.0473$. Multiple comparisons: $0.4136 \pm 0.1422, p=$ 0.0439; Fig. 8e and f). These laparotomy-induced changes were prevented by resistance exercise ( $\mathrm{p}-$ NR2A: $-0.2843 \pm 0.9373, p=0.0343$; p-NR2B: $-1.653 \pm 0.5286, p=0.0284$; p-AMPK-a: $-0.491 \pm 0.1422, p$ $=0.0146$; Fig. 8e-g), indicating resistance exercise prevented NMDA receptor-mediated excitotoxicity.

\section{Discussion}

Research into exercise to improve brain disorders is not novel but the use of "preconditioning" resistance training against PNDs is far and few between. This study showed that resistance exercise improves postoperative brain health from a number of standpoints, the most important of which is its effectiveness in aged animals. The elderly and those with $\mathrm{MCl}$ may have baseline deficits and neuroinflammatory 
tendencies that can be potentially reduced by this mode of training. It can confer cardiovascular as well as putative neurocognitive benefits perioperatively.

We have shown that compared with middle-aged counterparts, aged mice have increased body weight, higher basal levels of inflammatory markers, lower muscle strength and inferior aversive memory and hippocampal-dependent spatial learning. These risk factors for PNDs were reduced by a period of resistance exercise. Accordantly, the preoperative resistance exercise was found to improve surgeryinduced adverse effects including cognitive impairment, synaptic deficit and neuroinflammation. Further we report a novel finding regarding neuroinflammation and mitochondrial dysfunction that might form a vicious circle which ultimately may lead to synaptic dysfunction and cognitive dysfunction. This dysfunction can be relieved by resistance exercise, possibly by mediating the PGC1-a/BDNF pathway.

Pre-existing cognitive impairment is a major risk factor for PNDs that presents in $20 \%$ and $35 \%$ of elderly patients undergo total hip joint replacement ${ }^{31}$ and $C A B G{ }^{36}$, respectively. Consistent with previous reports 37 , we demonstrated pre-existing cognitive impairment in 18-month aged mice compared to middle-aged mice, and this pre-existing cognitive impairment was attenuated by resistance exercise (Fig. $1 \mathrm{~h}$ and $1 \mathrm{i}$ ). Moreover, postoperative cognitive decline was also prevented by resistance exercise pre-conditioning (Fig. 2e-f). Dendritic arborization and spine density are morphological features closely associated with synaptic plasticity, which underlies dynamic changes in neuronal circuits and is fundamental to learning and memory ${ }^{38}$. Resistance training prevented the postoperative decrease in dendritic process complexity and spine density in the DG of the hippocampus (Fig. 3), thus conferring benificial effect on perioperative cognitve function and synatic plasticity.

Increasing data revealed exaggerated neuroinflammation in aging ${ }^{39}$ and mild cognitive impairment $(\mathrm{MCl})^{40}$ contribute to the increased risk of PNDs. More importantly, elevated inflammatory cytokine levels correlate with the increased risk of cognitive decline with aging ${ }^{41}$ and progression from $M C l$ to $A D^{42}$. Our results suggest resistance exercise as a promising non-pharmacological approach to suppress both peripheral and neuroinflammation in aged mice, by reducing proinflammatory cytokines IL-1 $\beta$ and TNF-a in the liver and increasing the anti-inflammatory cytokine IL-10 in the hippocampus (Fig. 4a). Although we didn't reveal any change of IL-1 $\beta$, TNF- $\alpha$ and IL-10 on postoperative day 1 (which differs from several studies that showed significantly increased expression of IL-1 $\beta$ and TNF- ${ }^{43}$, but consistent with the data from our previous study ${ }^{22}$ ), resistance exercise was shown to reduce the elevated mRNA level of MCP-1 in the hippocampus following laparotomy (Fig. 4b). It has been suggested that astrocytes actively modulate immune responses in the CNS and evoking microglial activation via MCP-1-CCR2 signaling in surgery-induced cognitive dysfunction and neuroinflammation ${ }^{32}$. We demonstrated a prolonged neuroinflammation following surgery by showing proliferous and hypertrophic glia (Fig. 5).

Acute inflammatory response to insults results in either ultimate resolution of the inflammatory process, triggering cell regeneration and wound healing, or develops into prolonged inflammatory response that often leads to progressive cell and organ dysfunction. The anti-inflammatory effect of resistance exercise 
in this study can be attributed to the secretion of myokines from contracting muscles (Fig. 6a-c) and changes in body composition (Fig. 1d-e). The myokine FGF-21 promotes "browning" of white adipose tissue in mice in response to exercise ${ }^{44}$, thereby improving body composition and suppressing inflammation in obese individuals. Another myokine IL- 6 can reduce inflammation by inducing IL-10 and suppressing TNF-a production ${ }^{45}$. Adipose tissue secretes pro-inflammatory adipokines during physical inactivity and in metabolic disease ${ }^{46}$. The loss of visceral adipose tissue following exercise is IL-6 dependent ${ }^{47}$. Hence, myokines might mediate the anti-inflammatory effect of resistance exercise directly by modulating myokine secretion and indirectly by reducing the source of pro-inflammatory adipokines.

We demonstrated a significant increase of resistance exercise induced elevation of PGC-1a both in liver and hippocampus,PGC-1 $\mathrm{a}$ is one of the best characterized myokines that can induce hippocampal BDNF by regulating neuronal Fndc5 gene expression ${ }^{48}$. We also demonstrated an inhibition of BDNF/Akt/GSK$3 \beta$ signaling and an increased $\mathrm{Bax} / \mathrm{Bcl}-2$ ratio at 24 hours postop, which were attenuated by resistance exercise (Fig. 6d-f), and this effect on PGC-1a/BDNF signaling was still present at postoperative day 14 (Fig. 6g-h).

PGC-1a has been identified as a master regulator of mitochondrial biogenesis and the PGC-1a/BDNF pathway-mediated mitochondrial biogenesis and dynamic play important roles in the formation and maintenance of hippocampal dendritic spines and synapses ${ }^{49}$. Mitochondria are organelles in cells that widely known for their central role in supplying cellular energy ${ }^{50}$. In addition, mitochondria are involved in signal transduction, intracellular calcium regulation, and cellular differentiation, as well as regulating the cell growth and maintaining cell cycle. Recent studies indicated that mitochondria are vital in immune responses since they serve as both a target and source of innate immune signaling. Neuroinflammation has been shown to interfere with mitochondrial dynamic, membrane permeabilization, and mitophagy, resulting in mitochondrial dysfunction. Moreover, extracellular release of mitochondrial components exacerbates neuroinflammation, leading to a vicious inflammatory cycle and neuronal dysfunction. Hence preoperative resistance exercise mitigate post-surgical neuroinflammation through PGC-1a/BDNF pathway-mediated mitochondrial biogenesis and dynamic.

Evidence of deficits in mitochondrial biogenesis following surgery is supported by a reduced absolute mitochondrial number. Resistance exercise reduced the laparotomy-induced mitochondrial loss and mitochondrial damage, as indicated by the increased HSP60 in the cytosolic fraction and mitochondrial number, as well as decreased leakage of cytochrome $\mathrm{c}$ into cytosolic fraction percentage of damaged mitochondria (Fig. 7c-h). However, we did not find any changes in the expression of OXPHOS complexes $\mathrm{I}-\mathrm{V}$ in mitochondria fractions in the hippocampus (additional file 3 )

Mitochondrial biogenesis and mitophagy, as well as mitochondrial fission and fusion, contribute to the overall balance of mitochondria elimination and synthesis, which have been shown to be pivotal to the exercise-induced mitochondrial adaptations and neuronal benefits ${ }^{51}$. The ER-mitochondria contacts enable highly efficient transfer of $\mathrm{Ca}^{2+}$ from the ER to mitochondria, which is essential to maintain 
normal mitochondrial metabolism. However excessive ER-mitochondria $\mathrm{Ca}^{2+}$ influx will activate the mitochondrial permeability transition pore and induce leakage of cytochrome $\mathrm{c}$, thereby triggering cellar apoptosis ${ }^{52}$. Zheng et al have shown that DNA damage promotes the formation of ER-mitochondria contacts ${ }^{53}$, thus facilitating $\mathrm{Ca}^{2+}$ influx and DNA damage-induced apoptosis. Resistance exercise might be protective neurons in this regard as it reduced the formation of ER-mitochondria contacts in this experimental model (Fig. 8d). This hypothesis is supported by the post-surgical increase in mitofusin-2, a protein responsible for tether ER to mitochondria ${ }^{54}$, which was decreased by resistance exercise (Fig. 8b). However, the role of mitofusin- 2 in this process needs further exploration as not only is it involved in other important mitochondrial functions ${ }^{55}$ but also because of its controversial role in mediating ERmitochondria contacts in other pathophysiological conditions ${ }^{56,57}$.

Normal NMDA receptors activity in the hippocampus is essential to synaptic plasticity ${ }^{58}$. Early studies have reported that over-activation of NMDA receptors was closely associated with excitotoxicity and is implicated in many neurological diseases ${ }^{59,60}$. Subsequent evidence supports the "localization hypothesis" that indicated the dual nature of NMDA receptors based on their location ${ }^{61}$. Specifically, it has been proposed that the stimulation of synaptic NMDA receptors (mainly by upregulation of NR2B subunit) attribute to neuronal health and longevity ${ }^{61}$. Interestingly, we demonstrated a trend of losing synaptic NR2B following surgery, which was prevented by resistance exercise (Fig. 8f).

Proteomic analysis of tyrosine phosphorylation of synaptic glutamate receptors, including a-amino-3hydroxy-5-methyl-4-isoxazolepropionic acid (AMPA) receptors and NMDA receptors, found persistently upregulated NR2A-Y1246 and NR2B-Y1472 after brain ischemia ${ }^{62}$. Upregulation of NR2B-Y1472 was reported to be involved in excitotoxicity and contributed to brain injury ${ }^{63}$. Accordingly, we identified a significant increase in NR2A-Y1246 and NR2B-Y1472 after laparotomy, with preoperative resistance exercise preventing this rise (Fig. 8f). Along with these findings, activation of AMPK-a signaling, revealed by the upregulation of phosphorylated AMPK-a at Thr172, was found following surgery, that again was reduced by resistance exercise. The activation of AMPK under energy stress can be both neuroprotective ${ }^{64}$ and proapoptotic ${ }^{65}$. In stroke models, acute treatment with metformin, an AMPK-a agonist, has been shown to increase infarct volume; but reduce infarct volume with chronic administration ${ }^{66}$. Exercise has been well known to induce activation of AMPK signaling ${ }^{67}$. Given my exercise protocol lasted 5 weeks, the regulation of AMPK by resistance exercise in this study promoted neural survival, rather than neuronal death.

There are several limitations to this study, the main one of which is its largely descriptive as it was exploratory in nature. As resistance exercise seems to exert a wide range of benefits it was difficult to isolate a target for mechanistic study by way of pharmacological antagonists or knockout model. We are not sure if it deactivated a single upstream signal or conferred benefit from a multitude of factors released. Consequently, it is not possible to ascertain whether resistance exercise and improved postsurgical cognitive performance was epiphenomenal or mechanistically related. It was not possible to determine if the improved postoperative performance was consequential to a raised baseline that 
prevented it from sliding down to a critical point in the vicious cycle of neuroinflammation, or due to a protective effect that is independent of the baseline.

\section{Conclusions}

We provided experimental evidence for wide ranging and far-reaching benefits from a prehabilitative resistance exercise, especially with improving the reserve and resilience of mitochondria against detrimental effects of surgically induced neuroinflammation and synaptic deficits. Although the specific mechanisms remain to be elucidated, this approach can be evaluated as an interim non-pharmacological measure in clinical settings.

\section{Declarations}

Ethics approval and consent to participate: The experimental protocols were approved by the Faculty Committee on the Use of Live Animals in Teaching and Research (CULATR) in The University of Hong Kong (CULATR No. 4192-16).

Consent for publication: Not applicable

Availability of data and materials: The data that support the findings of this study are available from the corresponding author upon reasonable request.

Competing interests: The authors declared no conflict of or competing interests related to the studies.

Funding: This study was supported by the Seed Funding for Basic Research from the University of Hong Kong (grant numbers 201511159317 and 201910159171).

Authors' contributions: Yan Liu designed and performed the experiments, analyzed the data and drafted the manuscript. John Man Tak Chu designed primer that used in RT-PCR and revised the manuscript. You Ran participated in behavioral experiment and data analysis, Yan Zhang helped with blinding imaged and analyzing data. Raymond Chuen Chung Chang and Gordon Tin Chun Wong contributed to the experimental design, manuscript preparation and revision.

Acknowledgements: We would like to thank and acknowledge the professionalism and assistance from the Electron Microscopy Unit, Queen Mary Hospital, Hong Kong. Confocal imaging data were acquired using equipment maintained by the University of Hong Kong Li Ka Shing Faculty of Medicine Faculty Core Facility. The authors acknowledge the assistance of the University of Hong Kong Li Ka Shing Faculty of Medicine Faculty Core Facility

\section{Authors' information}

${ }^{1}$ Department of Anaesthesiology, LKS Faculty of Medicine, The University of Hong Kong, Pokfulam, Hong Kong SAR, China 
${ }^{2}$ Laboratory of Neurodegenerative Diseases, School of Biomedical Sciences, LKS Faculty of Medicine, The University of Hong Kong, Hong Kong SAR, China

${ }^{3}$ State Key Laboratory of Brain and Cognitive Sciences, The University of Hong Kong, Hong Kong SAR, China

${ }^{4}$ Department of Rehabilitation Medicine, The Third Affiliated Hospital of Sun Yat-Sen University, Guangzhou, China.

\section{References}

1. Bedford, P. D. Adverse cerebral effects of anaesthesia on old people. Lancet 269, 259-263 (1955).

2. Evered, L. et al. Recommendations for the nomenclature of cognitive change associated with anaesthesia and surgery-2018. Br J Anaesth 121, 1005-1012, doi:10.1016/j.bja.2017.11.087 (2018).

3. Deiner, S. \& Silverstein, J. H. Postoperative delirium and cognitive dysfunction. Br J Anaesth 103 Suppl 1, i41-46, doi:10.1093/bja/aep291 (2009).

4. Steinmetz, J., Christensen, K. B., Lund, T., Lohse, N. \& Rasmussen, L. S. Long-term consequences of postoperative cognitive dysfunction. Anesthesiology 110, 548-555, doi:10.1097/ALN.0b013e318195b569 (2009).

5. Evered, L. A., Silbert, B. S., Scott, D. A., Maruff, P. \& Ames, D. Prevalence of Dementia 7.5 Years after Coronary Artery Bypass Graft Surgery. Anesthesiology 125, 62-71, doi:10.1097/aln.0000000000001143 (2016).

6. Sprung, J. et al. Association of Mild Cognitive Impairment With Exposure to General Anesthesia for Surgical and Nonsurgical Procedures: A Population-Based Study. Mayo Clin Proc 91, 208-217, doi:10.1016/j.mayocp.2015.10.023 (2016).

7. Skvarc, D. R. et al. Post-Operative Cognitive Dysfunction: An exploration of the inflammatory hypothesis and novel therapies. Neurosci Biobehav Rev 84, 116-133, doi:10.1016/j.neubiorev.2017.11.011 (2018).

8. Bettio, L. E. B., Rajendran, L. \& Gil-Mohapel, J. The effects of aging in the hippocampus and cognitive decline. Neurosci Biobehav Rev 79, 66-86, doi:10.1016/j.neubiorev.2017.04.030 (2017).

9. Safavynia, S. A. \& Goldstein, P. A. The Role of Neuroinflammation in Postoperative Cognitive Dysfunction: Moving From Hypothesis to Treatment. Front Psychiatry 9, 752, doi:10.3389/fpsyt.2018.00752 (2018).

10. Bader, V. \& Winklhofer, K. F. Mitochondria at the interface between neurodegeneration and neuroinflammation. Semin Cell Dev Bio/ 99, 163-171, doi:10.1016/j.semcdb.2019.05.028 (2020).

11. Wilkins, H. M. \& Swerdlow, R. H. Relationships Between Mitochondria and Neuroinflammation: Implications for Alzheimer's Disease. Curr Top Med Chem 16, 849-857, doi:10.2174/1568026615666150827095102 (2016). 
12. Cibelli, M. et al. Role of interleukin-1 beta in postoperative cognitive dysfunction. Ann Neuro/ 68, 360368, doi:10.1002/ana.22082 (2010).

13. Hu, J. et al. Interleukin- 6 is both necessary and sufficient to produce perioperative neurocognitive disorder in mice. Br J Anaesth 120, 537-545, doi:10.1016/j.bja.2017.11.096 (2018).

14. Frederiksen, K. S., Gjerum, L., Waldemar, G. \& Hasselbalch, S. G. Effects of Physical Exercise on Alzheimer's Disease Biomarkers: A Systematic Review of Intervention Studies. J Alzheimers Dis 61, 359-372, doi:10.3233/jad-170567 (2018).

15. Mee-Inta, O., Zhao, Z.-W. \& Kuo, Y.-M. Physical Exercise Inhibits Inflammation and Microglial Activation. Cells 8, 691, doi:10.3390/cells8070691 (2019).

16. Mazur-Bialy, A. I., Bilski, J., Pochec, E. \& Brzozowski, T. New insight into the direct anti-inflammatory activity of a myokine irisin against proinflammatory activation of adipocytes. Implication for exercise in obesity. J Physiol Pharmacol 68, 243-251 (2017).

17. Ortega, E. The "bioregulatory effect of exercise" on the innate/inflammatory responses. $J$ Physiol Biochem 72, 361-369, doi:10.1007/s13105-016-0478-4 (2016).

18. Marques-Aleixo, I., Oliveira, P. J., Moreira, P. I., Magalhaes, J. \& Ascensao, A. Physical exercise as a possible strategy for brain protection: evidence from mitochondrial-mediated mechanisms. Prog Neurobio/ 99, 149-162, doi:10.1016/j.pneurobio.2012.08.002 (2012).

19. Yang, Z., Scott, C. A., Mao, C., Tang, J. \& Farmer, A. J. Resistance exercise versus aerobic exercise for type 2 diabetes: a systematic review and meta-analysis. Sports Med 44, 487-499, doi:10.1007/s40279-013-0128-8 (2014).

20. Liu, Y. et al. Short-term resistance exercise inhibits neuroinflammation and attenuates neuropathological changes in 3xTg Alzheimer's disease mice. J Neuroinflammation 17, 4, doi:10.1186/s12974-019-1653-7 (2020).

21. Deacon, R. M. Measuring the strength of mice. J Vis Exp, doi:10.3791/2610 (2013).

22. Huang, C., Chu, J. M., Liu, Y., Chang, R. C. \& Wong, G. T. Varenicline reduces DNA damage, tau mislocalization and post surgical cognitive impairment in aged mice. Neuropharmacology 143, 217227, doi:10.1016/j.neuropharm.2018.09.044 (2018).

23. Seibenhener, M. L. \& Wooten, M. C. Use of the Open Field Maze to measure locomotor and anxietylike behavior in mice. J Vis Exp, e52434, doi:10.3791/52434 (2015).

24. Antunes, M. \& Biala, G. The novel object recognition memory: neurobiology, test procedure, and its modifications. Cogn Process 13, 93-110, doi:10.1007/s10339-011-0430-z (2012).

25. Wan, Y. et al. Postoperative impairment of cognitive function in rats: a possible role for cytokinemediated inflammation in the hippocampus. Anesthesiology 106, 436-443, doi:10.1097/00000542200703000-00007 (2007).

26. Liu, Y., Wang, X. J., Wang, N., Cui, C. L. \& Wu, L. Z. Electroacupuncture Ameliorates Propofol-Induced Cognitive Impairment via an Opioid Receptor-Independent Mechanism. Am J Chin Med 44, 705-719, doi:10.1142/s0192415x16500385 (2016). 
27. Tavares, G. et al. Employing an open-source tool to assess astrocyte tridimensional structure. Brain Struct Funct 222, 1989-1999, doi:10.1007/s00429-016-1316-8 (2017).

28. Vincent, A. E. et al. The Spectrum of Mitochondrial Ultrastructural Defects in Mitochondrial Myopathy. Sci Rep 6, 30610, doi:10.1038/srep30610 (2016).

29. Fritz, N. E., McCarthy, C. J. \& Adamo, D. E. Handgrip strength as a means of monitoring progression of cognitive decline - A scoping review. Ageing Res Rev 35, 112-123, doi:10.1016/j.arr.2017.01.004 (2017).

30. Feinkohl, l., Winterer, G. \& Pischon, T. Obesity and post-operative cognitive dysfunction: a systematic review and meta-analysis. Diabetes Metab Res Rev 32, 643-651, doi:10.1002/dmrr.2786 (2016).

31. Silbert, B. et al. Preexisting cognitive impairment is associated with postoperative cognitive dysfunction after hip joint replacement surgery. Anesthesiology 122, 1224-1234, doi:10.1097/aln.0000000000000671 (2015).

32. Xu, J. et al. Astrocyte-derived CCL2 participates in surgery-induced cognitive dysfunction and neuroinflammation via evoking microglia activation. Behav Brain Res 332, 145-153, doi:10.1016/j.bbr.2017.05.066 (2017).

33. Mee-Inta, O., Zhao, Z. W. \& Kuo, Y. M. Physical Exercise Inhibits Inflammation and Microglial Activation. Cells 8, doi:10.3390/cells8070691 (2019).

34. Abd El-Kader, S. M. \& Al-Jiffri, O. H. Aerobic exercise improves quality of life, psychological well-being and systemic inflammation in subjects with Alzheimer's disease. Afr Health Sci 16, 1045-1055, doi:10.4314/ahs.v16i4.22 (2016).

35. Brunelle, J. K. \& Letai, A. Control of mitochondrial apoptosis by the Bcl-2 family. J Cell Sci 122, 437441, doi:10.1242/jcs.031682 (2009).

36. Hogue, C. W., Jr. et al. Preexisting cognitive impairment in women before cardiac surgery and its relationship with C-reactive protein concentrations. Anesth Analg 102, 1602-1608; table of contents, doi:10.1213/01.Ane.0000219591.10826.17 (2006).

37. Schonknecht, P., Pantel, J., Kruse, A. \& Schroder, J. Prevalence and natural course of agingassociated cognitive decline in a population-based sample of young-old subjects. Am J Psychiatry 162, 2071-2077, doi:10.1176/appi.ajp.162.11.2071 (2005).

38. Buffington, S. A., Huang, W. \& Costa-Mattioli, M. Translational control in synaptic plasticity and cognitive dysfunction. Annu Rev Neurosci 37, 17-38, doi:10.1146/annurev-neuro-071013-014100 (2014).

39. Youm, Y. H. et al. Canonical Nlrp3 inflammasome links systemic low-grade inflammation to functional decline in aging. Cell Metab 18, 519-532, doi:10.1016/j.cmet.2013.09.010 (2013).

40. Bradburn, S., Murgatroyd, C. \& Ray, N. Neuroinflammation in mild cognitive impairment and Alzheimer's disease: A meta-analysis. Ageing Res Rev 50, 1-8, doi:10.1016/j.arr.2019.01.002 (2019).

41. Atienza, M., Ziontz, J. \& Cantero, J. L. Low-grade inflammation in the relationship between sleep disruption, dysfunctional adiposity, and cognitive decline in aging. Sleep Med Rev 42, 171-183, doi:10.1016/j.smrv.2018.08.002 (2018). 
42. Brosseron, F., Krauthausen, M., Kummer, M. \& Heneka, M. T. Body fluid cytokine levels in mild cognitive impairment and Alzheimer's disease: a comparative overview. Mol Neurobio/ 50, 534-544, doi:10.1007/s12035-014-8657-1 (2014).

43. Zhang, X. et al. Activated brain mast cells contribute to postoperative cognitive dysfunction by evoking microglia activation and neuronal apoptosis. J Neuroinflammation 13, 127, doi:10.1186/s12974-016-0592-9 (2016).

44. Lee, P. et al. Irisin and FGF21 are cold-induced endocrine activators of brown fat function in humans. Cell Metab 19, 302-309, doi:10.1016/j.cmet.2013.12.017 (2014).

45. Petersen, A. M. \& Pedersen, B. K. The anti-inflammatory effect of exercise. J Appl Physiol (1985) 98, 1154-1162, doi:10.1152/japplphysiol.00164.2004 (2005).

46. Leal, L. G., Lopes, M. A. \& Batista, M. L., Jr. Physical Exercise-Induced Myokines and Muscle-Adipose Tissue Crosstalk: A Review of Current Knowledge and the Implications for Health and Metabolic Diseases. Front Physiol 9, 1307, doi:10.3389/fphys.2018.01307 (2018).

47. Wedell-Neergaard, A. S. et al. Exercise-Induced Changes in Visceral Adipose Tissue Mass Are Regulated by IL-6 Signaling: A Randomized Controlled Trial. Cell Metab 29, 844-855.e843, doi:10.1016/j.cmet.2018.12.007 (2019).

48. Wrann, C. D. et al. Exercise induces hippocampal BDNF through a PGC-1alpha/FNDC5 pathway. Cell Metab 18, 649-659, doi:10.1016/j.cmet.2013.09.008 (2013).

49. Cheng, A. et al. Involvement of PGC-1a in the formation and maintenance of neuronal dendritic spines. Nat Commun 3, 1250, doi:10.1038/ncomms2238 (2012).

50. Henze, K. \& Martin, W. Evolutionary biology: essence of mitochondria. Nature 426, 127-128, doi:10.1038/426127a (2003).

51. Twig, G. \& Shirihai, O. S. The interplay between mitochondrial dynamics and mitophagy. Antioxid Redox Signa/ 14, 1939-1951, doi:10.1089/ars.2010.3779 (2011).

52. Celsi, F. et al. Mitochondria, calcium and cell death: a deadly triad in neurodegeneration. Biochim Biophys Acta 1787, 335-344, doi:10.1016/j.bbabio.2009.02.021 (2009).

53. Zheng, P. et al. DNA damage triggers tubular endoplasmic reticulum extension to promote apoptosis by facilitating ER-mitochondria signaling. Cell Res 28, 833-854, doi:10.1038/s41422-018-0065-z (2018).

54. de Brito, O. M. \& Scorrano, L. Mitofusin 2 tethers endoplasmic reticulum to mitochondria. Nature 456, 605-610, doi:10.1038/nature07534 (2008).

55. Chen, H. et al. Mitofusins Mfn1 and Mfn2 coordinately regulate mitochondrial fusion and are essential for embryonic development. J Cell Biol 160, 189-200, doi:10.1083/jcb.200211046 (2003).

56. Wang, P. T. et al. Distinct mechanisms controlling rough and smooth endoplasmic reticulum contacts with mitochondria. J Cell Sci 128, 2759-2765, doi:10.1242/jcs.171132 (2015).

57. Filadi, R. et al. Mitofusin 2 ablation increases endoplasmic reticulum-mitochondria coupling. Proc Natl Acad Sci U S A 112, E2174-2181, doi:10.1073/pnas.1504880112 (2015). 
58. Watanabe, Y. et al. Age-Dependent Degeneration of Mature Dentate Gyrus Granule Cells Following NMDA Receptor Ablation. Front Mol Neurosci 8, 87, doi:10.3389/fnmol.2015.00087 (2015).

59. Cameron, H. A., McEwen, B. S. \& Gould, E. Regulation of adult neurogenesis by excitatory input and NMDA receptor activation in the dentate gyrus. J Neurosci 15, 4687-4692 (1995).

60. Deisseroth, K. et al. Excitation-neurogenesis coupling in adult neural stem/progenitor cells. Neuron 42, 535-552 (2004).

61. Zhou, X., Hollern, D., Liao, J., Andrechek, E. \& Wang, H. NMDA receptor-mediated excitotoxicity depends on the coactivation of synaptic and extrasynaptic receptors. Cell Death Dis 4, e560, doi:10.1038/cddis.2013.82 (2013).

62. Zhang, F., Guo, A., Liu, C., Comb, M. \& Hu, B. Phosphorylation and assembly of glutamate receptors after brain ischemia. Stroke 44, 170-176, doi:10.1161/strokeaha.112.667253 (2013).

63. Knox, R. et al. NR2B phosphorylation at tyrosine 1472 contributes to brain injury in a rodent model of neonatal hypoxia-ischemia. Stroke 45, 3040-3047, doi:10.1161/strokeaha.114.006170 (2014).

64. Weisova, P., Concannon, C. G., Devocelle, M., Prehn, J. H. \& Ward, M. W. Regulation of glucose transporter 3 surface expression by the AMP-activated protein kinase mediates tolerance to glutamate excitation in neurons. J Neurosci 29, 2997-3008, doi:10.1523/jneurosci.0354-09.2009 (2009).

65. Kilbride, S. M. et al. AMP-activated protein kinase mediates apoptosis in response to bioenergetic stress through activation of the pro-apoptotic Bcl-2 homology domain-3-only protein BMF. J Biol Chem 285, 36199-36206, doi:10.1074/jbc.M110.138107 (2010).

66. Li, J. \& McCullough, L. D. Effects of AMP-activated protein kinase in cerebral ischemia. J Cereb Blood Flow Metab 30, 480-492, doi:10.1038/jcbfm.2009.255 (2010).

67. Chrysostomou, V. et al. Exercise reverses age-related vulnerability of the retina to injury by preventing complement-mediated synapse elimination via a BDNF-dependent pathway. Aging Cell 15, 10821091, doi:10.1111/acel.12512 (2016).

\section{Figures}




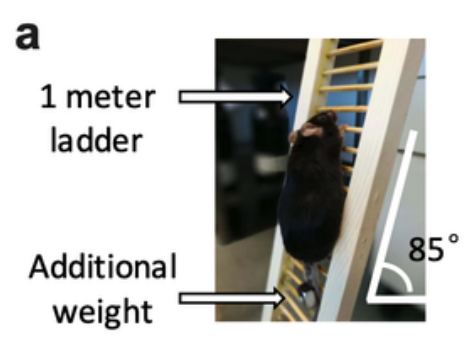

b
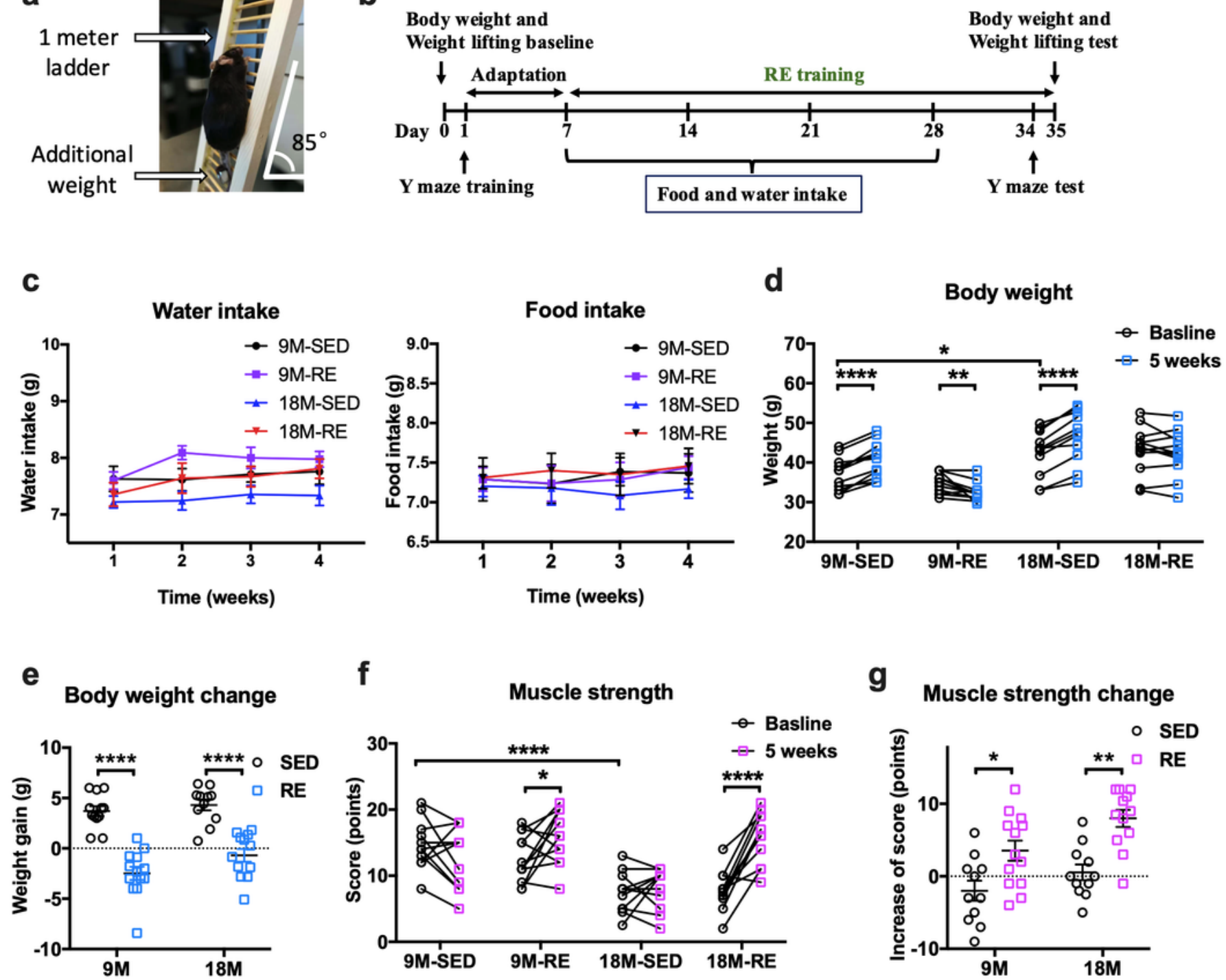

f

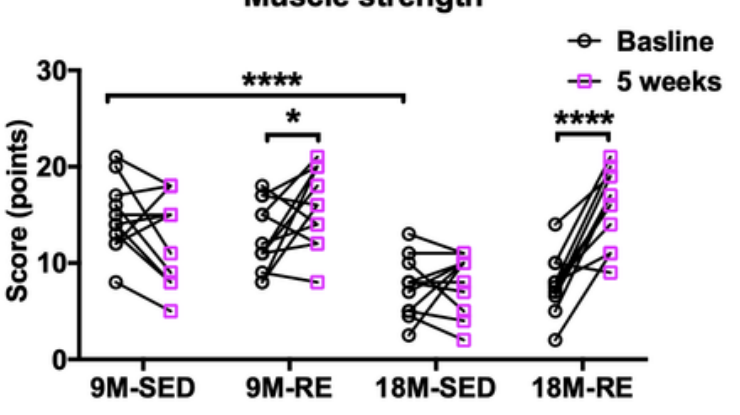

g Muscle strength change

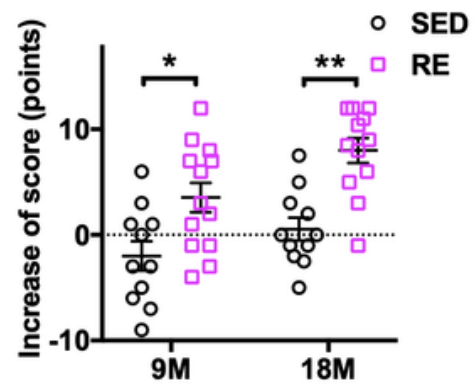

h Errors in Y maze test

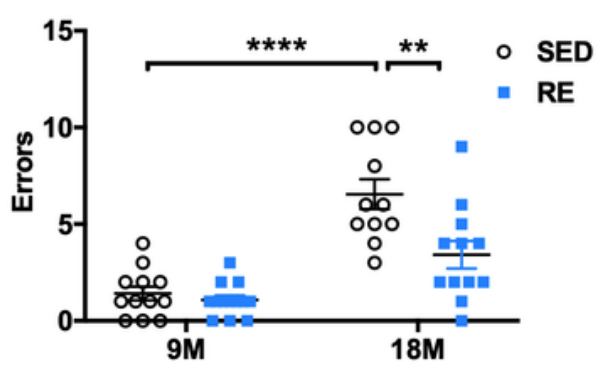

i Latency in $\mathrm{Y}$ maze test

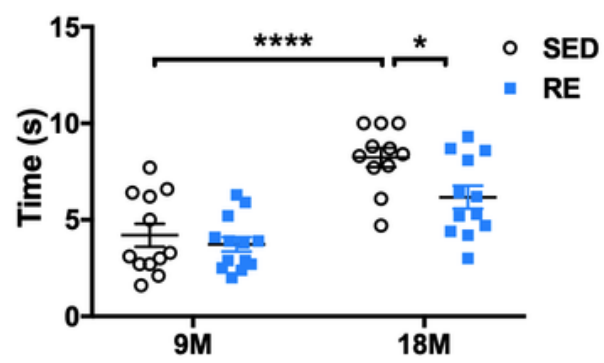

\section{Figure 1}

Effects of resistance exercise on general conditions and cognitive performance of 9 month and 18 month old mice (a) A picture of resistance exercise training apparatus. (b) Experimental design arrangements. (c) Water and food intake during the training period. Data represented averaged individual intake. Data analyzed by repeated-measures two-way ANOVA, followed by Bonferroni test as post-hoc comparisons. (d $\&$ e) Body weight and ( $\&$ \&) muscle strength before (baseline) and after resistance exercise. Data of 
body weight and muscle strength was analyzed by paired Student's t-tests, paired with pre-RE training (baseline); Data of body weight change and muscle strength change was analyzed by two-way ANOVA, followed by Tukey multiple comparisons test. Number of (h) errors and (i) escape latency from the $Y$ maze test. Data analyzed by two-way ANOVA, followed by Tukey multiple comparisons test. $\mathrm{n}=12$ for $9 \mathrm{M}$ SED, $n=13$ for $9 M-R E, n=11$ for $18 M-S E D$ and $n=12$ for $18 M-R E$. SED = sedentary group, RE = resistance exercise group. Data presented as mean \pm SEM. ${ }^{\star} p<0.05,{ }^{\star \star} \mathrm{p}<<0.01, \star \star \star \mathrm{p} \otimes 0.001,{ }^{\star \star \star *} \mathrm{p} \otimes 0.0001$. (except for muscle strength test, one mice in middle-aged exercise group suffered with a wound in its front paw in the testing day, therefore its data about muscle strength was excluded)

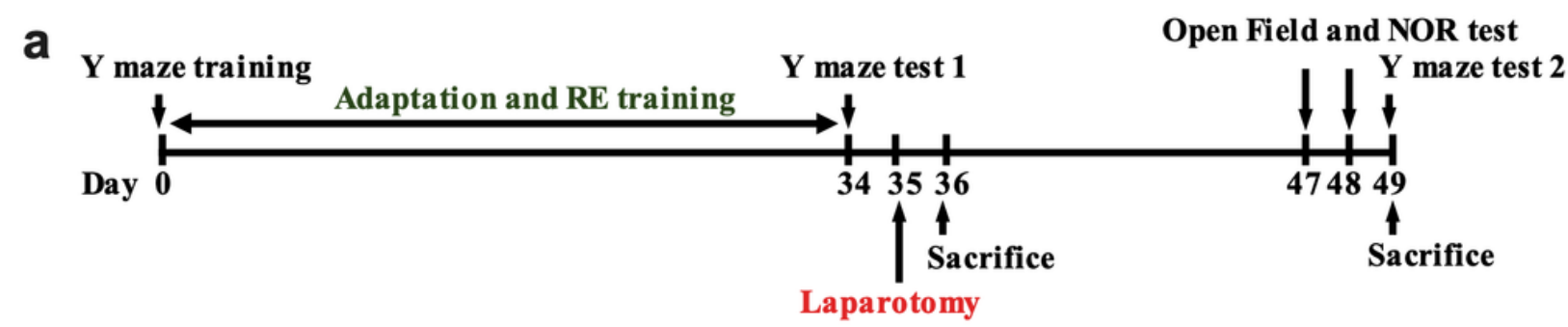

b

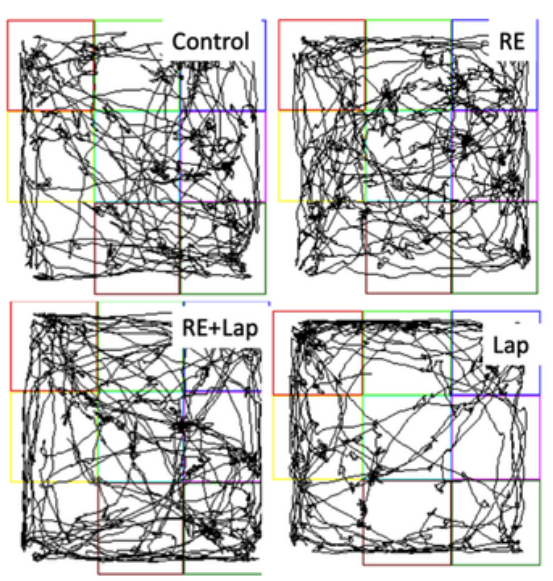

e

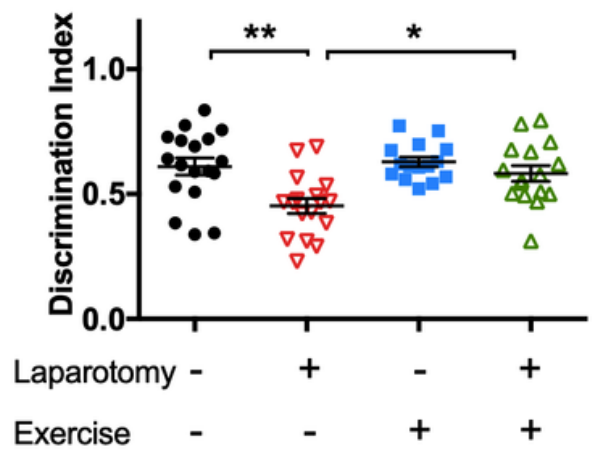

C

Open field (locomotor activity) $\mathbf{d}$

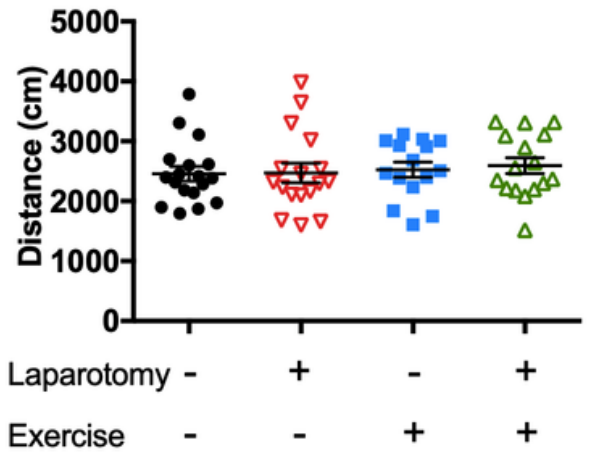

$\mathbf{f}$

Y-maze test (error)

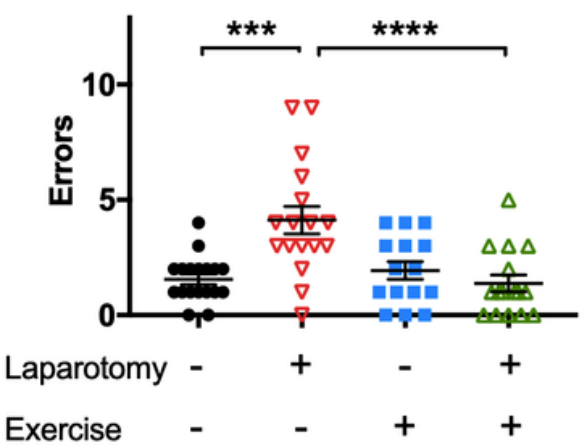

Open field (central time)

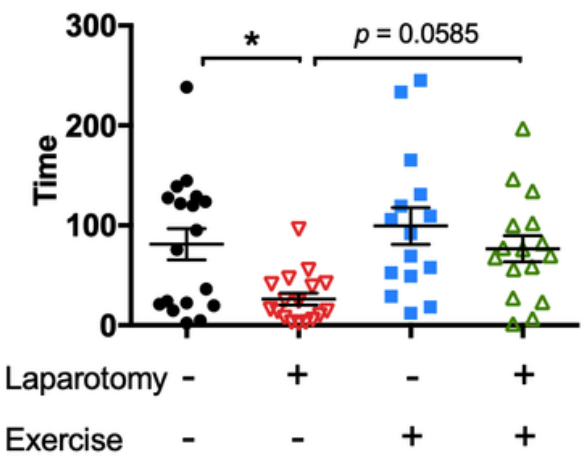

g

Y-maze test (latency )

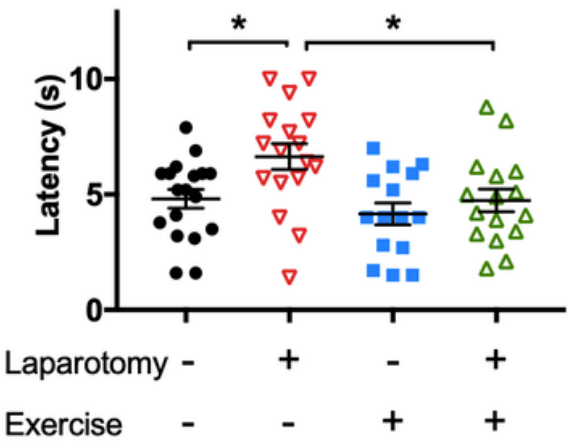

Figure 2

Effect of resistance training on postoperative cognitive performance of aged mice (a) Timeline showing the resistance exercise training and testing of postoperative cognitive performance relative to surgery. (b) Representative moving traces of mice in the open field arena. (c) Locomotor activity and (d) central 
duration in open field test. (e) Recognition index from novel object recognition test (Timenew/Timeold). (f) Escape latency and (g) number of errors in the Y-maze test. Data analyzed by two-way ANOVA, followed by Tukey multiple comparisons test. $n=18$ for control, $n=15$ for resistance exercise (RE) group, 16 for resistance exercise before laparotomy ( $R E+L a p)$ group and $n=17$ for laparotomy (Lap) group. Data presented as mean \pm SEM. ${ }^{*} p<0.05,{ }^{* \star} p<0.01$, ${ }^{\star * *} p<0.001$, ${ }^{* \star * *} p<0.0001$.

\section{Figure 3}

Resistance exercise preconditioning attenuated the decrease in dendritic process complexity and spine density following surgery (a) Representative photograph of Golgi staining of the hippocampus. (b) Representative photography of dendritic spines for the four experimental groups. (c) Quantitative analysis of spine density for the four experimental groups. Data analyzed by two-way ANOVA, followed by Tukey multiple comparisons test, $n=18$ in total, 4-5 dendrites were chosen at random from 4 mice in each group. (d-f) Sholl profiles generated with semi-automated analyses revealing process complexity of dendrites. Data analyzed by repeated-measures two-way ANOVA, followed by Bonferroni test as post-hoc comparisons, $n=14-15$ in total, 3-4 neurons were chosen from 4 mice in each group, Data presented as mean $\pm S E M, * p<0.05, * \star p<0.01$ and $* \star \star p<<0.001$, resistance exercise before laparotomy (RE+Lap) versus laparotomy (Lap) group. \#p $<0.05$ and \#\#p $<0.01$ Control versus laparotomy (Lap).

\section{a Immediate change of Pro- and anti-inflammatory Cytokines following RE}
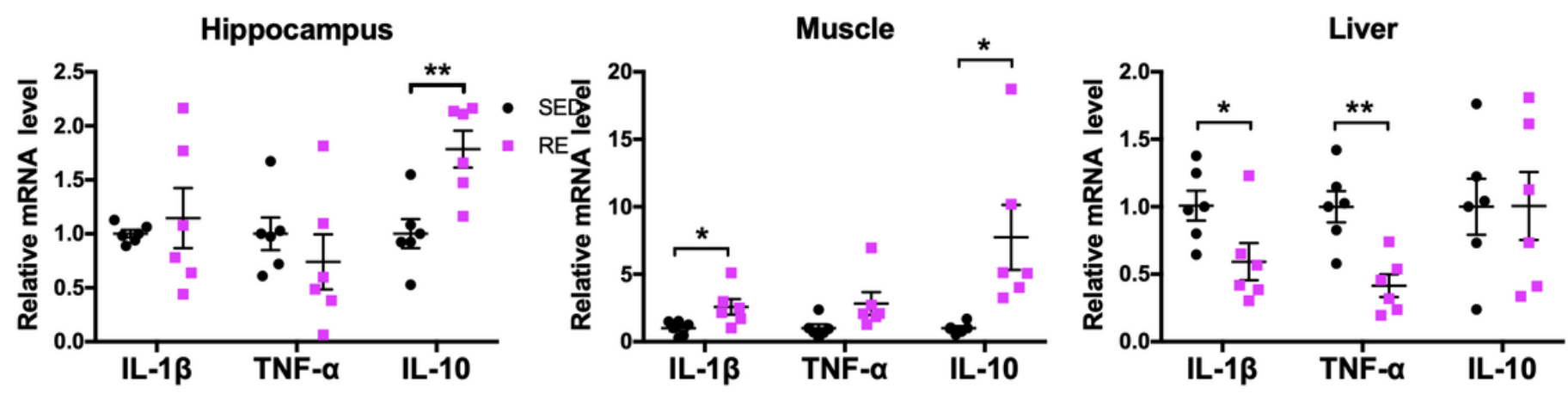

\section{b Early inflammatory response following laporomy}

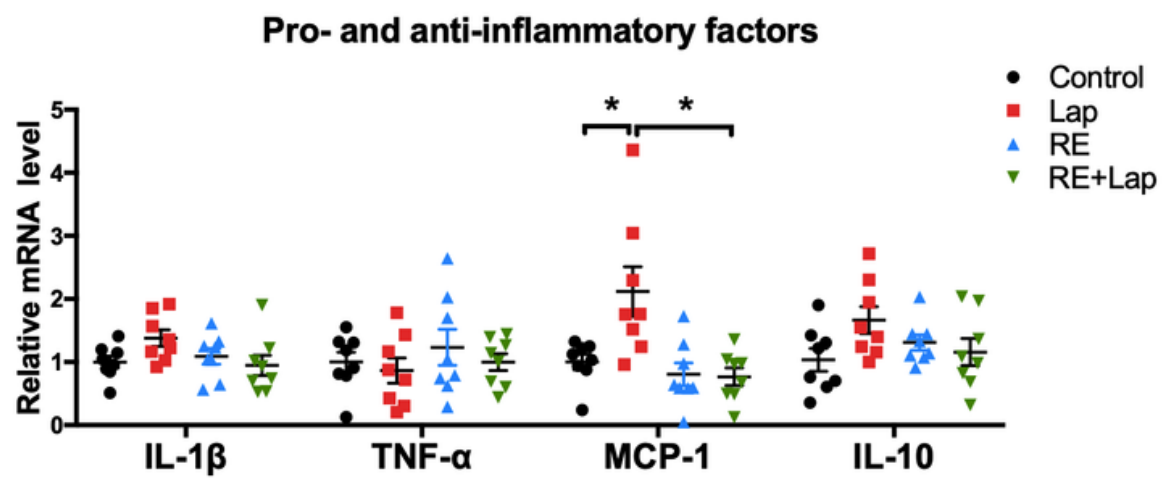




\section{Figure 4}

Effect of resistance training on acute inflammatory response (a) Relative mRNA levels of IL-1 $\beta$, TNF- $\alpha$ and IL-10 in the hippocampus, muscle and liver, $n=6$. (b) Relative mRNA levels of IL-1 $\beta$, TNF-a, MCP-1 and IL-10 in the hippocampus on postoperative 24 hours. Data analyzed by unpaired Student's t-test, $n=8$, Data presented as mean \pm SEM. ${ }^{\star} p<0.05,{ }^{\star \star} p<0.01$.

\section{Figure 5}

Prolonged inflammatory response in the hippocampus following resistance exercise and surgery (a) Iba1+ microglia and (b) Iba-1+ microglia body size. (c) Representative z-stacked confocal images showing Iba-1+ microglia (green) in the DG, CA1 and CA3 sub-regions of the hippocampus; Scale bar: $100 \mu \mathrm{m}$. (d) Representative z-stacked confocal images showed GFAP+ astrocytes (red) in the DG, CA1 and CA3 subregions of the hippocampus. Scale bar $=100 \mu \mathrm{m}$. (e) Quantitative analysis of fluorescence intensity of GFAP + astrocytes in the different hippocampal regions using Image J. (f) Representative zoomed in confocal images of GFAP+ astrocytes. Scale bar: $20 \mu \mathrm{m}$. (g) Count of primary processes from cell bodies of GFAP+ astrocyte. (h) GFAP+ astrocyte cell volume in different experimental groups. Data analyzed by two-way ANOVA, followed by Tukey multiple comparisons test, $n=8$ for RT-PCR examination, $n=5$ for IBA1 staining, 4 for GFAP staining, 20 for morphological analysis of astrocytes, Data presented as mean \pm SEM. ${ }^{*} p<0.05,{ }^{*} \mathrm{p}<0.01,{ }^{* \star} \mathrm{p}<0.001$. 

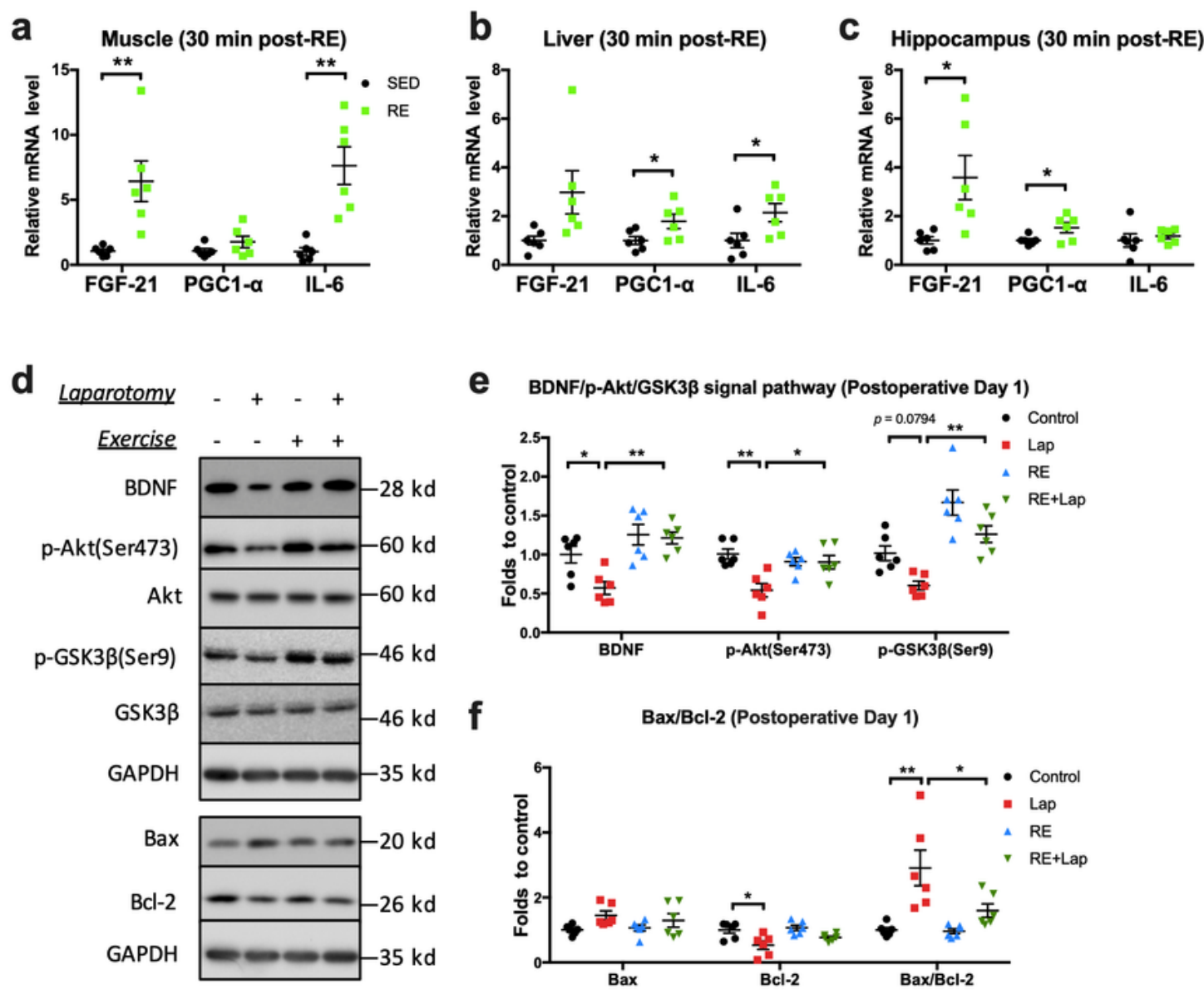

e BDNF/p-Akt/GSK3 $\beta$ signal pathway (Postoperative Day 1)
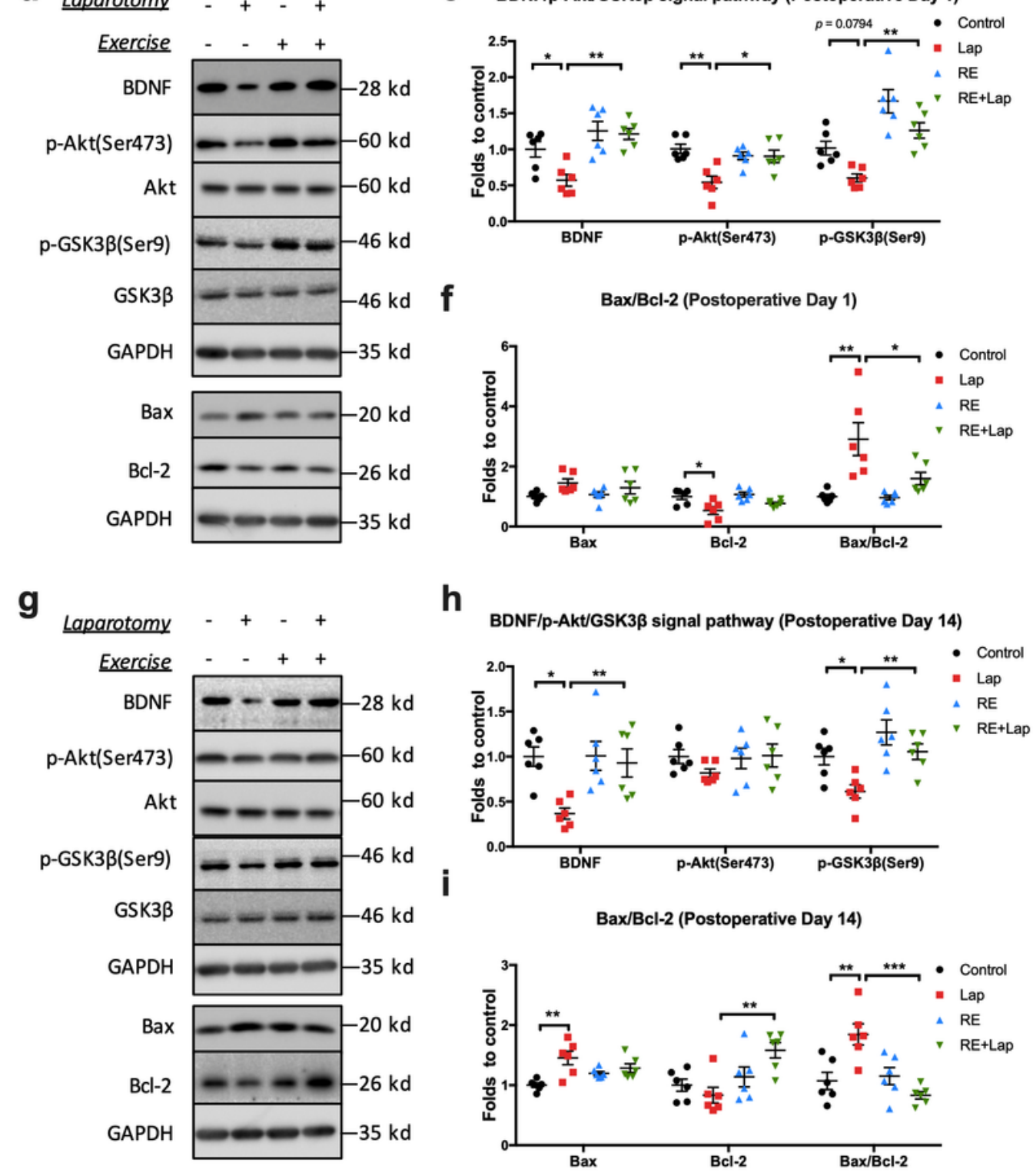

\section{Figure 6}

Changes in myokines and intracellular signal pathways following resistance exercise and surgery (a-c) Relative mRNA levels of FGF-21, PGC-1 $a$ and IL-6 in the hippocampus, muscle and liver. (d) Representative blots and (e-f) quantitative analysis of key proteins in intracellular signal transduction in the hippocampus at 24 hours postoperatively. (g) Representative blots and (h-j) quantitative analysis of key proteins in intracellular signal transduction in the hippocampus at 14 days postoperatively. The 
intensity of band of BDNF, Akt, GSK3ß囚Bax and Bcl-2 were normalized to that of GAPDH. Data analyzed by two-way ANOVA, followed by Tukey multiple comparisons test, $n=6$, Data presented as mean \pm SEM, ${ }^{*} p<0.05, * * p<0.01, * * * p<0.001$.

a

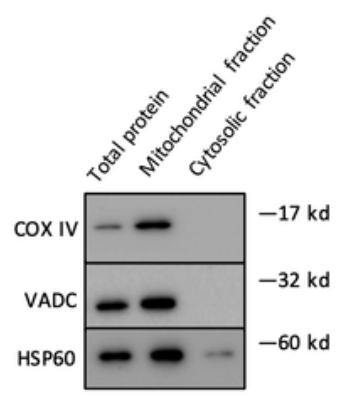

b

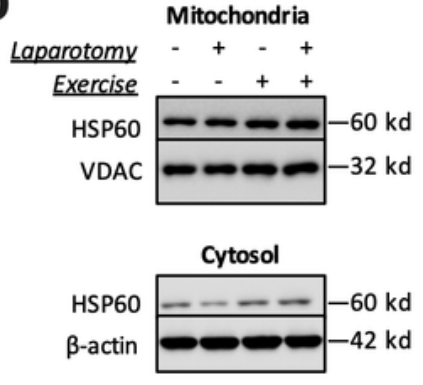

Expressions of HSP60

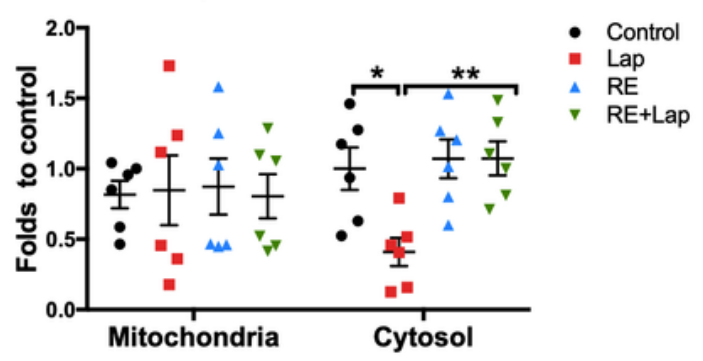

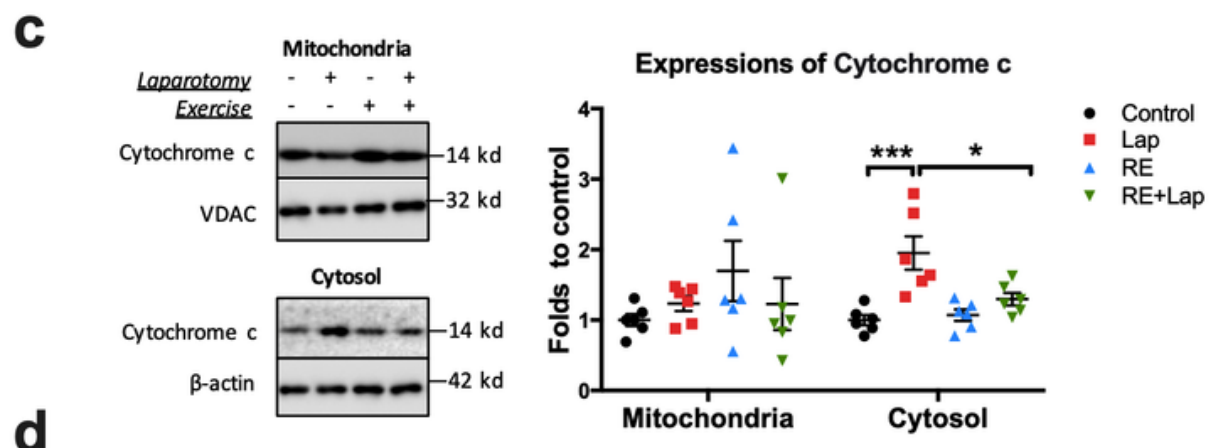

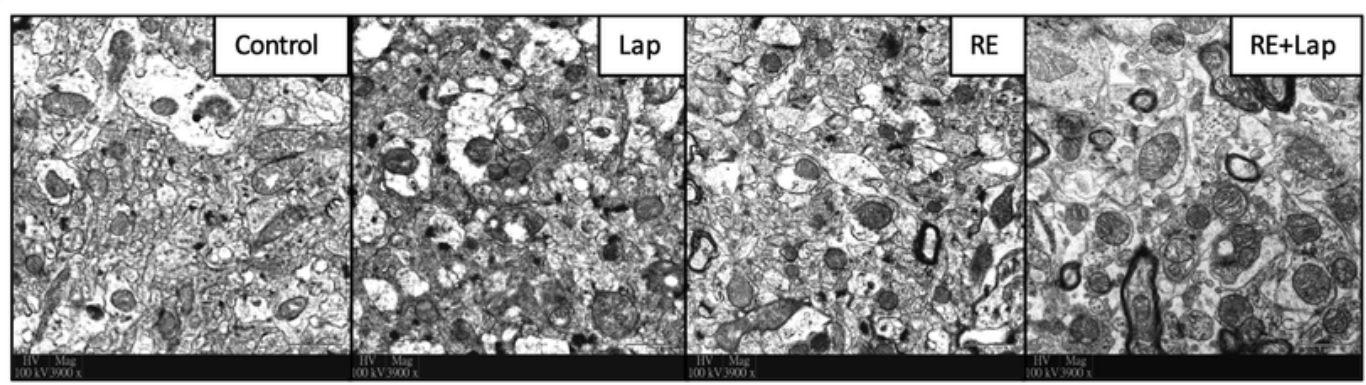

$\mathbf{f}$

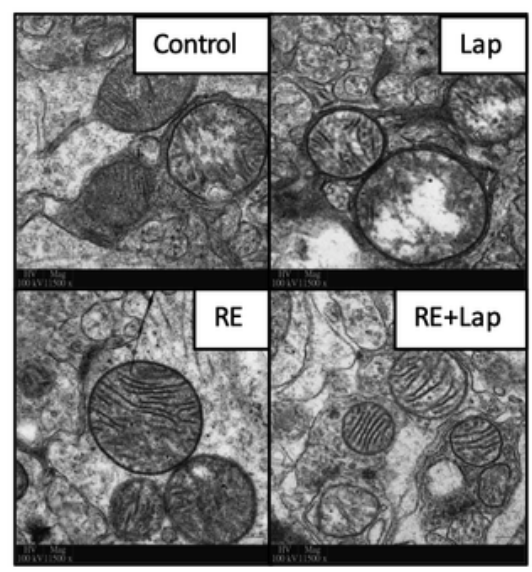

g

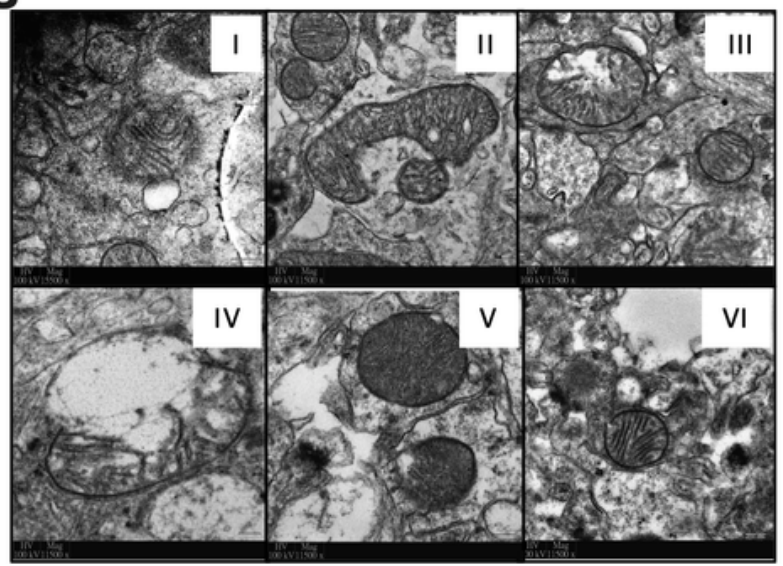

e

Number of mitochondria

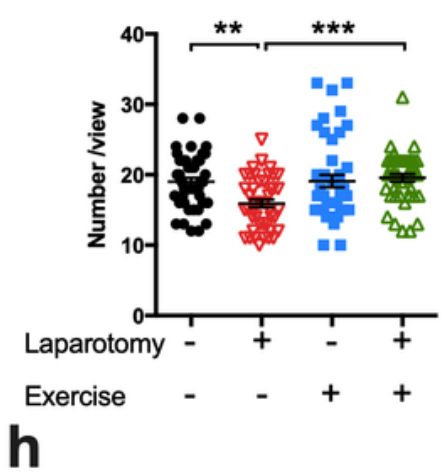

Abnormal mitochondria

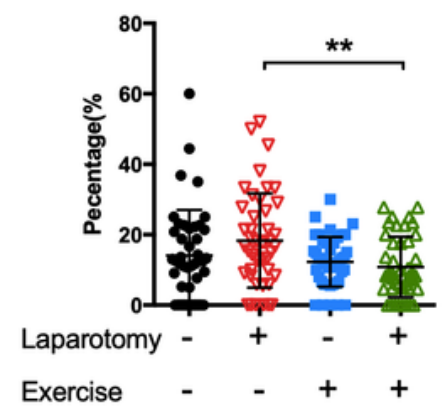

Figure 7

Changes in mitochondrial markers, density and morphology (a) Quality control of mitochondrial fraction isolation, equivalent amounts $(10 \mu \mathrm{g})$ of total protein, mitochondrial and cytosolic fractions were 
analyzed for mitochondrial enriched markers as indicated. (b) Representative blots and quantitative analysis of HSP60 in mitochondrial and cytosolic fractions, respectively. (c) Representative blots and quantitative analysis of cytochrome $c$ in mitochondrial and cytosolic fractions, respectively. The intensity of band was normalized to that of VDAC (mitochondrial fractions) or $\beta$-actin (cytosolic fractions). (d) Representative TEM pictures of mitochondria. (e) quantitative analysis of mitochondrial density. ( $\mathrm{f}$ and $\mathrm{g}$ ) Representative TEM pictures of abnormal mitochondria, including type I whirling cristae; type II swollen cristae; type III deficient cristae; type IV swollen outer membrane; type V sub-membrane vesicle and type VI discontinuous inter/outer membrane. (h) quantification of mitochondrial structural defects. Abnormal mitochondria with type I-VI have been expressed a percentage of the total pool of mitochondria. Data analyzed by two-way ANOVA, followed by Tukey multiple comparisons, for western blot: $n=6$; for TEM: $n=$ 44 comprise of 11 pictures from 4 mice in each group. Data are shown as a mean $\pm S E M$. ${ }^{\star} p<0.05$, $* * p<$ $0.01, * * * p<0.001, * * \star * p<0.0001$.

a

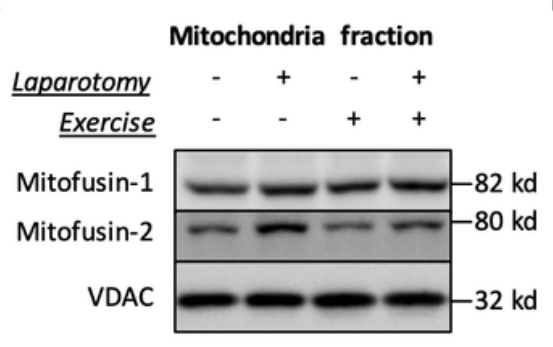

C
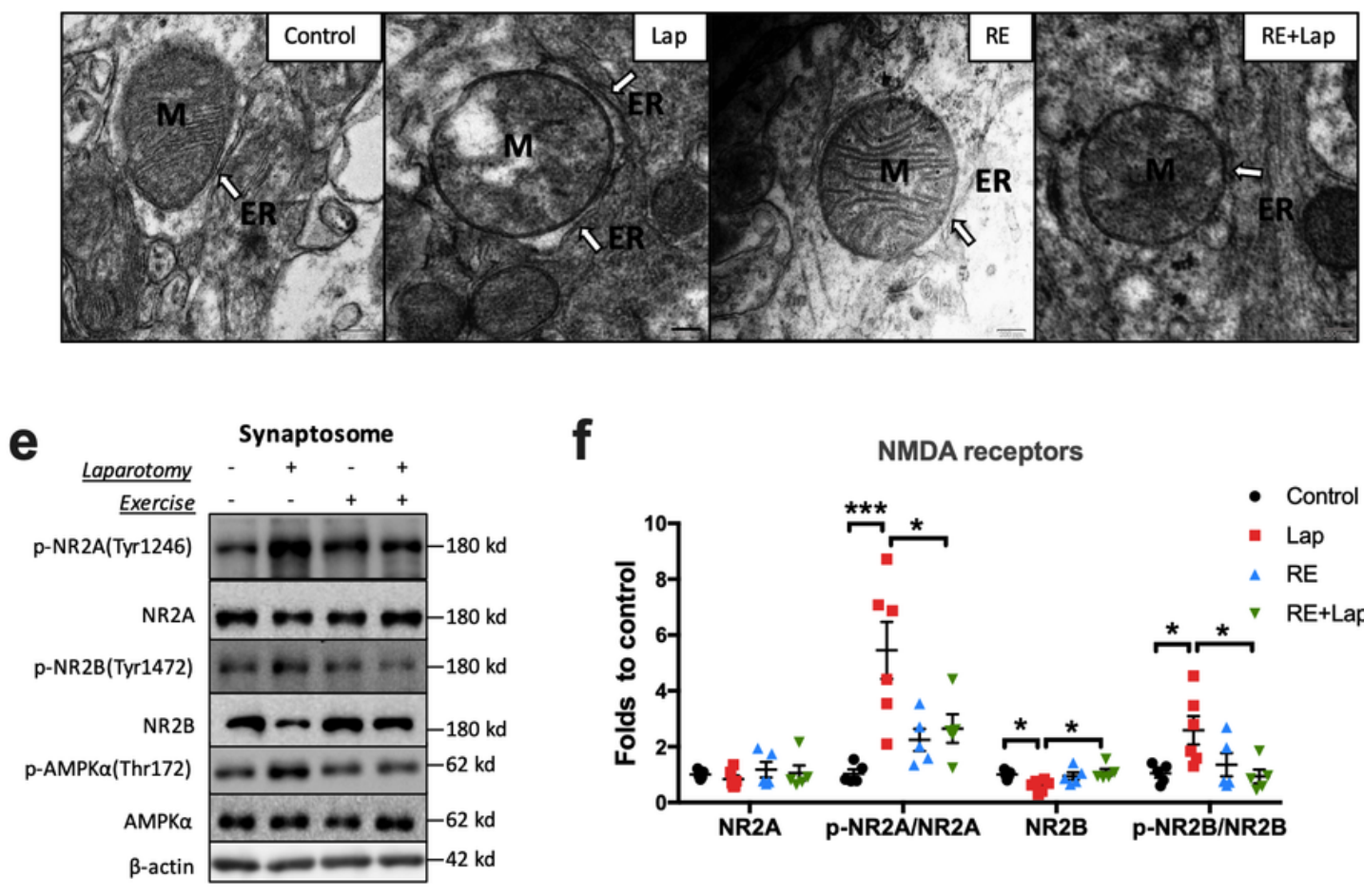

Mitochondrial dynamic proteins

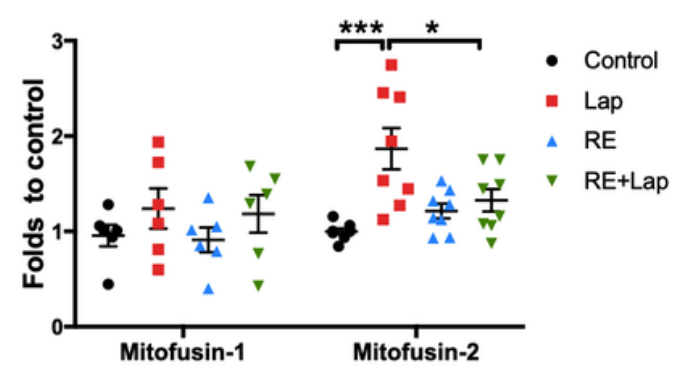

d

mitochondria-ER contact

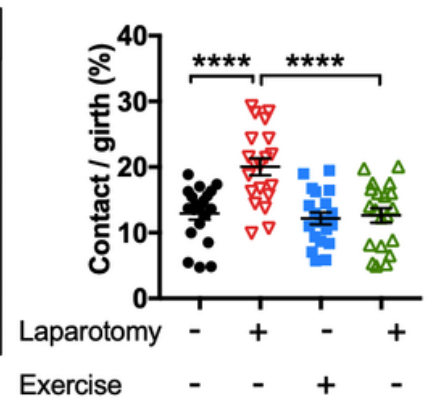

f

NMDA receptors

g

p-AMPK- $\alpha / A M P K-\alpha$

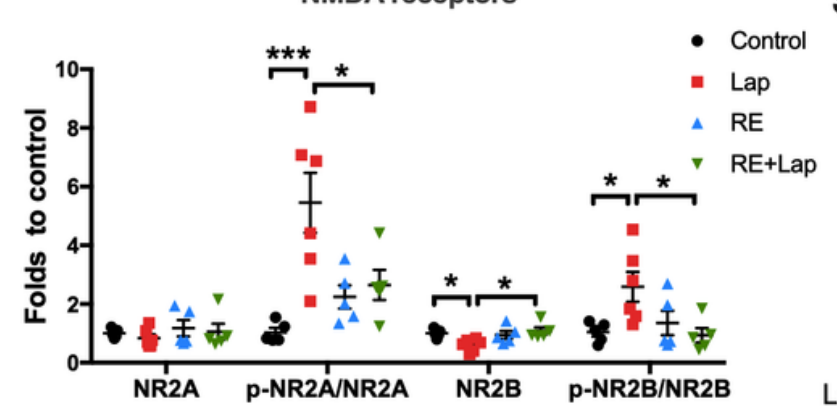

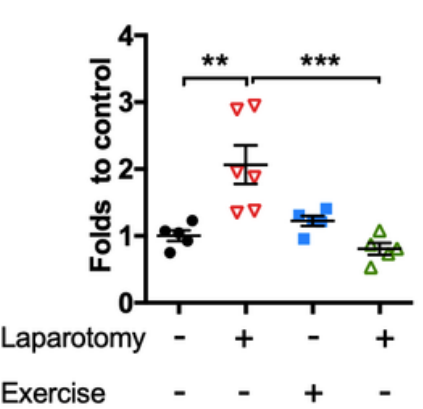

Figure 8 
Changes in mitochondria dynamic. ( $a$ and $b$ ) Representative blots and quantitative analysis of mitofusin1 and mitofusin- 2 in the mitochondria fraction, the intensity of band was normalized to that of VDAC. $n=6$ for mitofusin-1, 8 for mitofusin-2. (c) Representative TEM pictures of ER-mitochondria contacts, indicated by the white arrow. (d) Quantification of ER-mitochondria contacts, demonstrating by the contact area divided by the mitochondrial girth. $n=20$ comprised of 5 pictures from 4 mice in each group. (e-g) Representative blots and quantitative analysis of NMDARs and AMPK-a, with the intensity of band normalized to that of $\beta$-actin. Data analyzed by two-way ANOVA, followed by Tukey multiple comparisons test, $\mathrm{n}=5$ (except for Lap group, $\mathrm{n}=6$ for the expression of proteins in synatosome), Data presented as mean $\pm S E M,{ }^{\star} p<0.05,{ }^{\star \star} p<0.01,{ }^{\star \star \star} p<0.001$. M: mitochondria; ER: endoplasmic reticulum. $M=$ mitochondria; $E R=$ endoplasmic reticulum.

\section{Supplementary Files}

This is a list of supplementary files associated with this preprint. Click to download.

- Additionalfile118monthC57REtrainingvideo.mp4

- Additionalfile2.docx

- Additionalfile3.tiff 